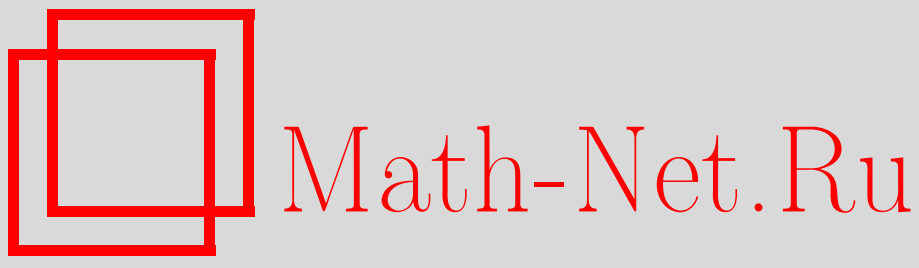

С. А. Назаров, Асимптотический анализ произвольно анизотропной пластины переменной толщины (пологой оболочки), Матем. сб., 2000, том 191, номер 7, 129 159

DOI: https://doi.org/10.4213/sm495

Использование Общероссийского математического портала Math-Net.Ru подразумевает, что вы прочитали и согласны с пользовательским соглашением

http: //www.mathnet.ru/rus/agreement

Параметры загрузки:

IP : 3.80 .181 .102

26 апреля 2023 г., $15: 33: 16$ 
УДК $517.946+539.3$

\author{
С. А. Назаров
}

\title{
Асимптотический анализ произвольно анизотропной пластины переменной толщины (пологой оболочки)
}

\begin{abstract}
Строятся главные члены асимптотики решения задачи теории упругости для тонкой пластины с искривленными основаниями, причем результирующая задача (двумерная модель) выписьвается в явной форме. Допускается произвольная анизотропия упругих свойств, которые к тому же могут зависеть от "быстрой" поперечной и "медленных" продольных переменных. Обоснование асимптотики проводится при помощи весового неравенства Корна. Отдельно обсуждаются случаи слоистых пластин, пологих оболочек и пластин с острьм краем.

Библиография: 51 название.
\end{abstract}

\section{§1. Постановка задачи и предварительное описание результатов}

1.1. Краевая задача. Пусть $\omega$ - область на плоскости $\mathbb{R}^{2}$, ограниченная простым гладким замкнутым контуром $\partial \omega$, и $H_{ \pm}-$гладкие в $\bar{\omega}$ функции, причем $H=H_{+}+H_{-}>0$. Через $h>0$ обозначим мальй параметр и введем тонкую трехмерную область

$$
\Omega_{h}^{0}=\left\{x=(y, z): y=\left(y_{1}, y_{2}\right) \in \omega,-h H_{-}(y)<z<h H_{+}(y)\right\}
$$

При этом $x$ и $y$ - декартовы координаты в пространстве и на плоскости $x_{3} \equiv$ $z=0$, а $\zeta=h^{-1} z$ - “быстрая" переменная, принадлежашая интервалу $\Upsilon(y)=$ $\left(-H_{-}(y), H_{+}(y)\right)$. Пусть еше $\mathscr{V}_{d} \subset \mathbb{R}^{2}-d$-окрестность контура $\partial \omega$ и $\Omega_{h} \subset \mathbb{R}^{3}-$ область, удовлетворяюшая условию

$$
\Omega_{h}^{0} \supset \Omega_{h} \supset\left\{x \in \Omega_{h}^{0}: y \in \omega \backslash \mathscr{V}_{c h}\right\}, \quad c>0 .
$$

Поверхность $\partial \Omega_{h}$ предполагаем кусочно гладкой (того же качества, что и $\partial \Omega_{h}^{0}$ ). Выделим на ней множество $\Gamma_{h} \subset\left\{x \in \partial \Omega_{h}: y \in \bar{\omega} \cap \mathscr{V}_{c h}\right\}$, расположенное около края пластины $\Omega_{h}$. Считаем, что

$$
\partial \Omega_{h} \backslash \Gamma_{h} \subset \Sigma_{+}^{0} \cup \Sigma_{-}^{0},
$$

где $\Sigma_{ \pm}^{0}=\left\{x: y \in \bar{\omega}, z= \pm h H_{ \pm}(y)\right\}$ - основания пластины (1.1).

Для того чтобы сформулировать задачу об изгибе упругой пластины $\Omega_{h}$ с зашемленной поверхностью $\Gamma_{h}$, договоримся интерпретировать вектор смешений $u$ 
как столбец $\left(u_{1}, u_{2}, u_{3}\right)^{t}(t-$ знак транспонирования) и образуем 6-столбец деформаций по формулам

$$
\begin{gathered}
\varepsilon(u)=D\left(\nabla_{x}\right)^{t} u \\
D\left(\nabla_{x}\right)=\left(\begin{array}{cccccc}
\partial_{1} & 0 & \alpha \partial_{2} & \alpha \partial_{z} & 0 & 0 \\
0 & \partial_{2} & \alpha \partial_{1} & 0 & \alpha \partial_{z} & 0 \\
0 & 0 & 0 & \alpha \partial_{1} & \alpha \partial_{2} & \partial_{z}
\end{array}\right), \quad \partial_{i}=\frac{\partial}{\partial y_{i}}, \quad \partial_{z}=\frac{\partial}{\partial z} .
\end{gathered}
$$

Столбец (1.4) содержит декартовы компоненты $\varepsilon_{11}, \varepsilon_{12}, \alpha^{-1} \varepsilon_{12}, \alpha^{-1} \varepsilon_{13}, \alpha^{-1} \varepsilon_{23}$, $\varepsilon_{33}$ тензора напряжений, а множители $\alpha=2^{-1 / 2}$ добавлены с целью уравнять естественные нормы столбца $\varepsilon(u)$ и симметричного тензора $\left(\varepsilon_{j k}\right)$. Согласно закону Гука вводится аналогичный (1.4) столбец напряжений

$$
\sigma(u)=A \varepsilon(u)=A D\left(\nabla_{x}\right)^{t} u .
$$

Здесь $A$-положительно определенная и симметрическая матрица-функция размером $6 \times 6$, аргументами которой назначим $y$ и $\zeta=h^{-1} z$. При помощи матрищы $A$ можно описать произвольные анизотропию и неоднородность пластины.

Во введенных обозначениях уравнения равновесия и краевые условия на свободных частях $\Sigma_{ \pm}=\left\{x \in \partial \Omega_{h} \backslash \bar{\Gamma}_{h}: x \in \Sigma_{ \pm}^{0}\right\}$ оснований пластины $\Omega_{h}$ выглядят следуюшим образом:

$$
\begin{aligned}
D\left(-\nabla_{x}\right) A D\left(\nabla_{x}\right)^{t} u & =f & \text { в } & \Omega_{h}, \\
D\left(\nu^{ \pm}\right) A D\left(\nabla_{x}\right)^{t} u & =g^{ \pm} & \text {на } & \Sigma_{ \pm}, \\
u & =0 & \text { на } & \Gamma_{h} .
\end{aligned}
$$

При этом $f$ и $g^{ \pm}$- векторы (столбцы) объемных сил и поверхностных усилий, a $\nu^{ \pm}$ - единичный вектор внешней нормали к $\Sigma_{ \pm}^{0}$ :

$$
\begin{gathered}
\nu^{ \pm}(h, y)=N_{ \pm}(h, y)^{-1}\left(-h \nabla_{y} H_{ \pm}(y), \pm 1\right)^{t}, \\
N_{ \pm}(h, y)=\left(1+h^{2}\left|\nabla_{y} H_{ \pm}(y)\right|^{2}\right)^{1 / 2}, \quad \nabla_{y}=\left(\partial_{1}, \partial_{2}\right)^{t} .
\end{gathered}
$$

Если $u$ и $v$ - гладкие вектор-функции, причем $v$ удовлетворяет условию Дирихле (1.9), то верна формула Грина

$$
\begin{aligned}
& \left(D\left(-\nabla_{x}\right) A D\left(\nabla_{x}\right)^{t} u, v\right)_{\Omega_{h}}+\sum_{ \pm}\left(D\left(\nu^{ \pm}\right) A D\left(\nabla_{x}\right)^{t} u, v\right)_{\Sigma_{ \pm}} \\
& \quad=2 E\left(u, v ; \Omega_{h}\right) \equiv\left(A D\left(\nabla_{x}\right)^{t} u, D\left(\nabla_{x}\right)^{t} v\right)_{\Omega_{h}},
\end{aligned}
$$

где $(\cdot, \cdot) \Xi-$ скалярное произведение в $L_{2}(\Xi)$, а функционал $E\left(u, u ; \Omega_{h}\right) \geqslant 0$ имеет смысл упругой энергии, запасенной телом $\Omega_{h}$; благодаря положительной определенности $A$

$$
E\left(u, u ; \Omega_{h}\right) \geqslant c_{A}\left\|D\left(\nabla_{x}\right)^{t} u ; L_{2}\left(\Omega_{h}\right)\right\|^{2} .
$$

Через $\stackrel{\circ}{H}{ }^{1}\left(\Omega_{h}, \Gamma_{h}\right)^{3}$ обозначим пространство вектор-функций из соболевского класса $H^{1}\left(\Omega_{h}\right)^{3}$, удовлетворяюших краевому условию (1.9) (верхний индекс 3 указывает количество компонент). Известно (см., например, [1]-[4]) неравенство Корна

$$
\left\|u ; H^{1}\left(\Omega_{h}\right)\right\|^{2} \leqslant c_{h} E\left(u, u ; \Omega_{h}\right) \quad \forall u \in \stackrel{\circ}{H^{1}}\left(\Omega_{h}, \Gamma_{h}\right)^{3},
$$

которое обеспечивает разрешимость рассматриваемой задачи. 
ПреДЛОЖЕнИЕ 1.1. При любъх $f \in L_{2}\left(\Omega_{h}\right)^{3}$ u $g^{ \pm} \in L_{2}\left(\Sigma_{ \pm}\right)^{3}$ существует единственное (обобщенное) решение $u \in \stackrel{\circ}{H}^{1}\left(\Omega_{h}, \Gamma_{h}\right)^{3}$ задачи (1.7)-(1.9).

ЗАмЕчАниЕ 1.2. Требования к правым частям $f$ и $g^{ \pm}$, разумеется, можно ослабить, чем мы и воспользуемся в $\S 3$.

Константа $c_{h}>0$ в (1.13) сушественно зависит от области $\Omega_{h}$, а значит, и от малого геометрического параметра $h$. Для обоснования асимптотического представления решения задачи (1.7)-(1.9), являюшегося основной целью работы, потребуется уточнить неравенство Корна (1.13), сделав его весовым (см. §4).

1.2. Обрисовка результатов. Основой двумерных моделей тонких пластин, используемых в конкретных инженерных расчетах, служит классическая гипотеза прямых нормалей, предложенная Кирхгофом в 1850 г., имеюшая чисто физическую природу и потому придающая моделям формальный и эвристический характер. Строго обоснованной можно считать лиш модель цилиндрической однородной изотропной пластины, для которой помимо оценок погрешностей (см. [5]-[10]) разработана техника пограничных слоев, позволяюшая конструировать асимптотические решения с произвольной, наперед заданной точностью (см. [11]-[19], а также книги $[20 ;$ гл. 15,16$]$ и $[21 ; \S 5.7])$. Настоящая статья значительно расширяет объем результатов технической теории, получивших строгое математическое обоснование, и даже содержит анализ ситуаций, не затронутых прикладными исследованиями. Именно, при помощи обшего алгорифма изучения эллиптических краевых задач в тонких областях, развитого в [9], [14], [22] (см. также [20; гл. 16]), в $\S 2$ определяются главные члены асимптотики решения задачи (1.7)-(1.9), т.е. выводится результируюшая задача для произвольно анизотропной и неоднородной искривленной пластины при единственном ограничении: физические и геометрические характеристики плавно меняются в продольных направлениях (нет быстрой осцилляции; ср. с [22]-[24]). Обоснование найденной асимптотики проводится в $\S 3$ при помощи весового неравенства Корна, установленного в 4 для пластин с переменной толщиной и обобщающего прежние результаты [6], [25]-[27]. Подчеркнем, что доказанные оценки являются асимптотически точными и в некотором смысле коэрцитивными - в мажорантах явно выделена зависимость от составляющих правых частей задачи (1.7)-(1.9), а ухудшение их дифференциальных свойств снижает гарантированный порядок точности.

В случае цилиндрических пластин $\omega \times(-h / 2, h / 2)$ построенные в $\S 2$ двумерные модели не отличаются от известных ранее в технических теориях (см., например, [28]) или найденных путем формального асимптотического анализа (см. [29], [30]). Тем самьм, настоящая статья дает косвенное, математическое подтверждение гипотез Кирхгофа.

Явление пограничного слоя здесь не затрагивается, хотя включение соответствуюшего члена в асимптотическое приближение позволяет установить поточечные, а не интегральные оценки остатка (в [31] это было сделано для тонких стержней). Дело в том, что в согласии с общими принципами построения пограничных слоев, изложенными в [14] и [20; гл. 16], слабое искривление границы не привносит каких-либо осложнений, поскольку существует почти тождественный диффеоморфизм окрестности $\mathscr{V}_{c h^{1 / 2}}$ на себя, спрямляюший поверхности $\Sigma_{ \pm}^{0} \cap \mathscr{V}_{c h^{1 / 2}}$, т.е. 
дополнительно может потребоваться лишш разложение коэффициентов возникших дифференциальных операторов в ряды Тейлора по переменной $\operatorname{dist}\{y, \partial \omega\}$. Сказанное не касается случая пластины с острым краем, для которой результирующая система вырождается на $\partial \omega$ (см. [32], [33; $\S 37])$, а пограничные слои описываются решениями задач в параболических областях (см. [34]-[36]) или вовсе исчезают.

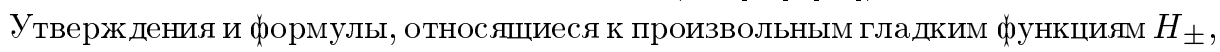
легко приспосабливаются к частной, но важной форме пологой оболочки, для которой толшина $H(y)$ лишш слагаемым $O\left(h^{2}\right)$ отличается от постоянной. В связи с этой интерпретацией пластины переменной толщины упомянем статьи [37], [38], в которых доказываются теоремы о сходимости пространственных полей смешений и напряжений к решениям двумерных уравнений пологих оболочек. Как недавно стало известно автору, асимптотический анализ такой оболочки из однородного изотропного материала проведен М. Дож, Ж. Андреою, Е. Фау (M. Dauge, G. Andreoiu, E. Faou).

\section{§ 2. Формальные асимптотические конструкции}

2.1. Разложения операторов. Вводя быструю переменную $\zeta=h^{-1} z$ и учитывая формулу (1.10), получаем

$$
\begin{aligned}
& D\left(\nabla_{x}\right)=h^{-1} \mathbb{D}_{\zeta}+\mathbb{D}_{y}, \quad D\left(\nu^{ \pm}\right)=-N_{ \pm}^{-1}\left( \pm \mathbb{D}_{1}-h \mathbb{D}_{ \pm}\right), \\
\mathbb{D}_{y}= & \left(\begin{array}{cccccc}
\partial_{1} & 0 & \alpha \partial_{2} & 0 & 0 & 0 \\
0 & \partial_{2} & \alpha \partial_{1} & 0 & 0 & 0 \\
0 & 0 & 0 & \alpha \partial_{1} & \alpha \partial_{2} & 0
\end{array}\right), \quad \mathbb{D}_{1}=\left(\begin{array}{cccccc}
0 & 0 & 0 & \alpha & 0 & 0 \\
0 & 0 & 0 & 0 & \alpha & 0 \\
0 & 0 & 0 & 0 & 0 & 1
\end{array}\right), \\
\mathbb{D}_{ \pm}= & \left(\begin{array}{cccccc}
\partial_{1} H_{ \pm} & 0 & \alpha \partial_{2} H_{ \pm} & 0 & 0 & 0 \\
0 & \partial_{2} H_{ \pm} & \alpha \partial_{1} H_{ \pm} & 0 & 0 & 0 \\
0 & 0 & 0 & \alpha \partial_{1} H_{ \pm} & \alpha \partial_{2} H_{ \pm} & 0
\end{array}\right), \quad \mathbb{D}_{\zeta}=\mathbb{D}_{1} \partial_{\zeta} .
\end{aligned}
$$

Таким образом, матричные дифференциальные операторы $L$ и $B^{ \pm}$из левых частей равенств (1.7) и (1.8) допускают расшепления

$$
\begin{gathered}
L\left(h, x, \nabla_{x}\right)=h^{-2} L^{0}\left(y, \zeta, \partial_{\zeta}\right)+h^{-1} L^{1}\left(y, \zeta, \nabla_{y}, \partial_{\zeta}\right)+h^{0} L^{2}\left(y, \zeta, \nabla_{y}\right), \\
N_{ \pm}(h, y) B^{ \pm}\left(h, y, \nabla_{x}\right)=h^{-1} B^{0 \pm}\left(y, \partial_{\zeta}\right)+h^{0} B^{1 \pm}\left(y, \nabla_{y}, \partial_{\zeta}\right)+h^{1} B^{2 \pm}\left(y, \nabla_{y}\right),
\end{gathered}
$$

в которых

$$
\begin{aligned}
& L^{0}=-\mathbb{D}_{\zeta} A \mathbb{D}_{\zeta}^{t}, \quad L^{1}=-\mathbb{D}_{\zeta} A \mathbb{D}_{y}^{t}-\mathbb{D}_{y} A \mathbb{D}_{\zeta}^{t}, \quad L^{2}=-\mathbb{D}_{y} A \mathbb{D}_{y}^{t}, \\
& B^{0 \pm}= \pm \mathbb{D}_{1} A \mathbb{D}_{\zeta}^{t}, \quad B^{1 \pm}= \pm \mathbb{D}_{1} A \mathbb{D}_{y}^{t}-\mathbb{D}_{ \pm} A \mathbb{D}_{\zeta}^{t}, \quad B^{2 \pm}=-\mathbb{D}_{ \pm} A \mathbb{D}_{y}^{t} \text {. }
\end{aligned}
$$

Обращаем внимание на следующее ключевое тождество, используемое на протяжении всей статьи:

$$
\mathbb{D}_{y}^{t} e^{3}=\mathbb{D}_{1}^{t}\left(e^{1} \frac{\partial}{\partial y_{1}}+e^{2} \frac{\partial}{\partial y_{2}}\right)
$$

здесь $e^{j}-$ орт оси $x_{j}$. 
2.2. Асимптотические анзацы. Предположим, что правые части задачи (1.7)-(1.9) допускают представления

$$
\begin{aligned}
f(h, x) & =h^{-1} f^{0}(y, \zeta)+h^{0} \tilde{f}^{0}(y)+\widetilde{f}(h, x), \\
g^{ \pm}(h, y) & =h^{0} g^{0 \pm}(y)+\widetilde{g}(h, x),
\end{aligned}
$$

причем их члены подчинены требованиям

$$
\begin{gathered}
\int_{\Upsilon(y)} f_{3}^{0}(y, \zeta) d \zeta+g_{3}^{0+}(y)+g_{3}^{0-}(y)=0, \quad y \in \omega, \\
\bar{f}_{1}^{0}=\bar{f}_{2}^{0}=0 .
\end{gathered}
$$

В этом параграфе функции $f^{0}, \bar{f}^{0}$ и $g^{0 \pm}$ считаем гладкими, а правильные ограничения накладываем на них и на остатки $\widetilde{f}, \widetilde{g}$ в п. 3.2 .

Решение задачи (1.7)-(1.9) с правыми частями (2.5) ишем в виде суммы

$$
h^{-2} U^{-2}(y, \zeta)+h^{-1} U^{-1}(y, \zeta)+h^{0} U^{0}(y, \zeta)+h^{1} U^{1}(y, \zeta)+\cdots,
$$

два первых члена которой выглядят следуюшим образом:

$$
U^{-2}(y, \zeta)=e^{3} w_{3}(y), \quad U^{-1}(y, \zeta)=\sum_{i=1}^{2} e^{i}\left(w_{i}(y)-\zeta \frac{\partial w_{3}}{\partial y_{i}}(y)\right)
$$

Столбец $w=\left(w_{1}, w_{2}, w_{3}\right)^{t}$ подлежит дальнейшему определению. Функция $w_{3}$ описывает в главном прогиб пластины, а $w_{1}$ и $w_{2}$ - ее продольные смещения.

ЗАмЕчАниЕ 2.1. Используется тот же асимптотический анзац (2.7), (2.8), что и в случае "прямых" пластин $\omega \times(-h / 2, h / 2)$. Имеется много путей его объяснения как механического толка [28], [29], так и математического [6]-[9], [22]. Расхождение показателей степеней $h$ при $w_{3}$ и $w_{1}, w_{2}$ отражает очевидный факт: пластину легче изогнуть, чем растянуть. То же касается и представления (2.5), поскольку благодаря (2.6) поперечные силы имеют порядок $h^{1}$, а продольные - порядок $h^{0}$. Любую правую часть можно представить в виде $(2.5),(2.6)$ после умножения на $h^{m} \mathrm{c}$ подходяшим $m$.

2.3. Предельные задачи. Выделяя в (2.2) старшие (относительно $h$ ) части операторов, приходим к задаче Неймана на отрезке $\Upsilon(y)=\left(-H_{-}(y), H_{+}(y)\right)$ с параметром $y \in \omega$

$$
L^{0} U=F \quad \text { на } \Upsilon(y), \quad B^{0 \pm} U=G^{ \pm} \text {при } \zeta= \pm H_{ \pm}(y) .
$$

Очередное утверждение известно и очевидно. 
ЛЕмма 2.2. Задача (2.9) с правыми частями $F \in L_{2}(\Upsilon(y))^{3}, G^{ \pm} \in \mathbb{R}^{3}$ имеет решение $U \in H^{2}(\Upsilon(y))^{3}$ в том и только том случае, если выполнень условия разрешимости

$$
\int_{\Upsilon(y)} F_{k}(y, \zeta) d \zeta+G_{k}^{+}(y)+G_{k}^{-}(y)=0, \quad k=1,2,3
$$

Решение $U$ определено с точностью до постоянного (по z) слагаемого, но будучи подчинено условиям ортогональности

$$
\int_{\Upsilon(y)} U_{k}(y, \zeta) d \zeta=0, \quad k=1,2,3,
$$

становится единственным, удовлетворяет оценке

$$
\left\|U ; H^{2}(\Upsilon(y))\right\| \leqslant c\left(\left\|F ; L_{2}(\Upsilon(y))\right\|+\left|G^{+}\right|+\left|G^{-}\right|\right)
$$

и наследует от $F$ и $G^{ \pm}$гладкую зависимость относительно переменных $y=\left(y_{1}, y_{2}\right) \in \omega$.

Подставим разложения $(2.7),(2.5)$ и (2.3) в уравнения $(1.7),(1.8)$ и, собрав коэффициенты при одинаковых степенях параметра $h$, получим набор предельных задач:

$$
\begin{gathered}
L^{0} U^{j}=-L^{1} U^{j-1}-L^{2} U^{j-2}+\delta_{j, 1} f^{0} \equiv F^{j} \quad \text { на } \Upsilon(y) \\
B^{0 \pm} U^{j}=-B^{1 \pm} U^{j-1}-B^{2 \pm} U^{j-2}+\delta_{j, 1} g^{0 \pm} \equiv G^{j \pm} \quad \text { при } \quad \zeta= \pm H_{ \pm}(y) .
\end{gathered}
$$

Здесь $j=-2,-1,0,1$ и $U^{k}=0$ при $k<-2$.

Задача (2.13) при $j=-2$ однородная, и поэтому функция $U^{-2}$ из $(2.8)$, не зависящая от $\zeta$, является ее решением. Непосредственной проверкой нетрудно убедиться, что в силу тождества (2.4) справедливо равенство

$$
\mathbb{D}_{\zeta}^{t} U^{-1}+\mathbb{D}_{y}^{t} U^{-2}=0
$$

обеспечивающее выполнение (2.13) с $j=-1$. При учете $(2.13),(2.14)$ и (2.3) находим, что

$$
\begin{gathered}
F^{0}=\mathbb{D}_{\zeta} A \mathbb{D}_{y}^{t} U^{-1}+D_{y} A\left\{\mathbb{D}_{\zeta}^{t} U^{-1}+\mathbb{D}_{y}^{t} U^{-2}\right\}=\mathbb{D}_{\zeta} A \mathbb{D}_{y}^{t} U^{-1} \\
G^{0 \pm}=\mp \mathbb{D}_{1} A \mathbb{D}_{y}^{t} U^{-1}+\mathbb{D}_{ \pm} A\left\{\mathbb{D}_{\zeta}^{t} U^{-1}+\mathbb{D}_{y}^{t} U^{-2}\right\}=\mp \mathbb{D}_{1} A \mathbb{D}_{y}^{t} U^{-1} .
\end{gathered}
$$

Заметим, что для любой функции $\mathscr{W} \in H^{1}(\Upsilon(y))^{6}$

$$
\begin{aligned}
\int_{\Upsilon(y)}\left(e^{j}\right)^{t} \mathbb{D}_{\zeta} \mathscr{W}(\zeta) d \zeta+\left(e^{j}\right)^{t} & \mathbb{D}_{1}\left(\mathscr{W}\left(H_{+}(y)\right)-\mathscr{W}\left(-H_{-}(y)\right)\right) \\
& =\int_{\Upsilon(y)}\left(\mathbb{D}_{\zeta}^{t} e^{j}\right)^{t} \mathscr{W}(\zeta) d \zeta=0, \quad j=1,2,3 .
\end{aligned}
$$


Теперь, взяв $\mathscr{W}=A \mathbb{D}_{y}^{t} U^{-1}$, видим, что правые части (2.15) удовлетворяют условиям (2.10), т.е. задача (2.13) при $j=0$ разрешима. Кроме того, манипулируя с выражениями (2.1) и (2.8), выводим, что

$$
\mathscr{D}\left(\nabla_{y}\right)=\left(\begin{array}{cccccc}
\partial_{1} & 0 & \alpha \partial_{2} & 0 & 0 & 0 \\
0 & \partial_{2} & \alpha \partial_{1} & 0 & 0 & 0 \\
0 & 0 & 0 & \alpha \partial_{1}^{2} & \alpha \partial_{2}^{2} & \partial_{1} \partial_{2}
\end{array}\right), \quad \mathscr{Y}(\zeta)=\left(\begin{array}{cc}
\mathbf{1}_{3} & -\alpha^{-1} \zeta \mathbf{1}_{3} \\
\mathbf{0}_{3} & \mathbf{0}_{3}
\end{array}\right) ;
$$

здесь $\mathbf{1}_{q}$ и $\mathbf{0}_{q}-$ единичная и нулевая матрищы размером $q \times q$. Итак, решение $U^{0}$ представимо следующим образом:

$$
U^{0}(y, \zeta)=\mathscr{X}(y, \zeta) \mathscr{D}\left(\nabla_{y}\right)^{t} w(y)
$$

При этом матрица $\mathscr{X}$ имеет размеры $3 \times 6$ и удовлетворяет равенствам

$$
-\mathbb{D}_{\zeta} A \mathbb{D}_{\zeta}^{t} \mathscr{X}=\mathbb{D}_{\zeta} A \mathscr{Y} \text { на } \Upsilon(y), \quad \pm \mathbb{D}_{1} A \mathbb{D}_{\zeta}^{t} \mathscr{X}=\mp \mathbb{D}_{1} A \mathscr{Y} \text { при } \zeta= \pm H_{ \pm}(y)
$$

Иными словами, столбцы $\mathscr{X}^{1}, \ldots, \mathscr{X}^{6}$ матрицы $\mathscr{X}$ являются решениями задачи (2.9) со специальными правыми частями. Подчиним эти столбцы условиям ортогональности (2.11) - по лемме 2.2 матрица $\mathscr{X}$ будет гладко зависеть от $y \in \bar{\omega}$.

2.4. Вывод результирующей задачи. Перейдем к рассмотрению задачи (2.13) при $j=1$, для чего понадобится следующее тождество, выражающее правило дифференцирования интегралов с переменными пределами:

$$
\int_{\Upsilon(y)} \mathbb{D}_{y} \mathscr{W}(y, \zeta) d \zeta+\sum_{ \pm} \mathbb{D}_{ \pm} \mathscr{W}\left(y, \pm H_{ \pm}(y)\right)=\mathbb{D}_{y} \int_{\Upsilon(y)} \mathscr{W}(y, \zeta) d \zeta
$$

Благодаря этому тождеству и предположению (2.6) правые части

$$
\begin{aligned}
F^{1} & =\mathbb{D}_{\zeta} A \mathbb{D}_{y}^{t} U^{0}+\mathbb{D}_{y} A\left\{\mathbb{D}_{\zeta}^{t} U^{0}+\mathbb{D}_{y}^{t} U^{-1}\right\}+f^{0}, \\
G^{1 \pm} & =\mp \mathbb{D}_{1} A \mathbb{D}_{y}^{t} U^{0}+\mathbb{D}_{ \pm} A\left\{\mathbb{D}_{\zeta}^{t} U^{0}+\mathbb{D}_{y}^{t} U^{-1}\right\}+g^{0 \pm}
\end{aligned}
$$

удовлетворяют условию (2.10), $k=3$. В самом деле, используя дополнительно (2.16) и (2.4), находим, что

$$
\begin{aligned}
\int_{\Upsilon(y)} & F_{3}^{1}(y, \zeta) d \zeta+G_{3}^{1+}(y)+G_{3}^{1-}(y) \\
= & \left(e^{3}\right)^{t}\left[\int_{\Upsilon(y)} \mathbb{D}_{y} A\left\{\mathbb{D}_{\zeta}^{t} U^{0}+\mathbb{D}_{y}^{t} U^{-1}\right\} d \zeta\right. \\
& \left.+\left.\sum_{ \pm} \mathbb{D}_{ \pm} A\left\{\mathbb{D}_{\zeta}^{t} U^{0}+\mathbb{D}_{y}^{t} U^{-1}\right\}\right|_{\zeta= \pm H_{ \pm}(y)}\right] \\
= & \left(e^{3}\right)^{t} \mathbb{D}_{y} \int_{\Upsilon(y)} A\left\{\mathbb{D}_{\zeta}^{t} U^{0}+\mathbb{D}_{y}^{t} U^{-1}\right\} d \zeta
\end{aligned}
$$




$$
\begin{aligned}
= & \sum_{i=1}^{2}\left(e^{i}\right)^{t} \frac{\partial}{\partial y_{i}} \int_{\Upsilon(y)} \mathbb{D}_{1} A\left\{\mathbb{D}_{\zeta}^{t} U^{0}+\mathbb{D}_{y}^{t} U^{-1}\right\} d \zeta \\
= & \sum_{i=1}^{2} \frac{\partial}{\partial y_{i}} \int_{\Upsilon(y)}\left(\mathbb{D}_{\zeta}^{t} \zeta e^{i}\right)^{t} A\left\{\mathbb{D}_{\zeta}^{t} U^{0}+\mathbb{D}_{y}^{t} U^{-1}\right\} d \zeta \\
= & -\sum_{i=1}^{2} \frac{\partial}{\partial y_{i}}\left(e^{i}\right)^{t}\left[\int_{\Upsilon(y)} \zeta \mathbb{D}_{\zeta} A\left\{\mathbb{D}_{\zeta}^{t} U^{0}+\mathbb{D}_{y}^{t} U^{-1}\right\} d \zeta\right. \\
& \left.+\sum_{ \pm} \pm\left.\left(\zeta \mathbb{D}_{1} A\left\{\mathbb{D}_{\zeta}^{t} U^{0}+\mathbb{D}_{y}^{t} U^{-1}\right\}\right)\right|_{\zeta= \pm H_{ \pm}(y)}\right]=0 .
\end{aligned}
$$

Последнее равенство возникло в результате уточнения соотношений (2.13) с $j=0$ при помоши формул (2.3) и (2.15).

Для правых частей (2.21) условия разрешимости (2.10) при $k \equiv i=1,2$ записываются в виде

$$
\begin{aligned}
0 & =\left(e^{i}\right)^{t}\left[\int_{\Upsilon(y)} \mathbb{D}_{y} A \mathscr{Z} d \zeta+\left.\sum_{ \pm} \mathbb{D}_{ \pm} A \mathscr{Z}\right|_{\zeta= \pm H_{ \pm}(y)}\right] \mathscr{D}\left(\nabla_{y}\right)^{t} w+\mathscr{F}_{i} \\
& =\left(e^{i}\right)^{t} \mathbb{D}_{y} \int_{\Upsilon(y)} A \mathscr{Z} d \zeta \mathscr{D}\left(\nabla_{y}\right)^{t} w+\mathscr{F}_{i}, \quad i=1,2,
\end{aligned}
$$

где в соответствии с $(2.17)$ и (2.18)

$$
\begin{gathered}
\mathbb{D}_{\zeta}^{t} U^{0}+\mathbb{D}_{y}^{t} U^{-1}=\mathscr{Z} \mathscr{D}\left(\nabla_{y}\right)^{t} w, \quad \mathscr{Z}(y, \zeta)=\mathbb{D}_{1}^{t} \partial_{\zeta} \mathscr{X}(y, \zeta)+\mathscr{Y}(\zeta), \\
\mathscr{F}_{i}(y)=\int_{\Upsilon(y)} f_{i}^{0}(y, \zeta) d \zeta+g_{i}^{0+}(y)+g_{i}^{0-}(y), \quad i=1,2 .
\end{gathered}
$$

Итак, образовалась пара дифференциальных уравнений (2.23), в которых фигурирует до сих пор неизвестньй столбец $w$. Еще одно уравнение появится при дальнейшем асимптотическом анализе, а полученная система уравнений, называемая результирующей, послужит для определения $w$, т.е. для конкретизации членов $(2.8),(2.18)$ анзаца (2.7).

Недостающее уравнение можно определить при рассмотрении задачи для очередного члена $U^{2}$. Однако в приближенное решение, используемое в п. 3.3 , не войдет даже слагаемое $U^{1}$ (это принципиальньй момент, позволяющий максимально ослабить требования к гладкости правых частей - здесь мы занимаемся построением и обоснованием только главных членов асимптотик). Поэтому более логично вывести упомянутое уравнение при помощи иных соображений. Именно обработаем невязку, оставляемую в системе (1.7) суммой $\mathbf{U}$ четырех слагаемых (2.7). При учете (2.2) и (2.5) имеем

$$
\begin{aligned}
L \mathbf{U}-f= & h^{-4} L^{0} U^{-2}+h^{-3}\left(L^{0} U^{-1}+L^{1} U^{-2}\right) \\
& +h^{-2}\left(L^{0} U^{0}+L^{1} U^{-1}+L^{2} U^{-2}\right)+h^{-1}\left(L^{0} U^{1}+L^{1} U^{0}+L^{2} U^{-1}-f^{0}\right) \\
& +h^{0}\left(L^{1} U^{1}+L^{2} U^{0}-\bar{f}^{0}\right)+h^{1} L^{2} U^{1}-\widetilde{f} .
\end{aligned}
$$


Векторы $U^{-2}, \ldots, U^{1}$ были выбраны так, чтобы аннулировать множители при $h^{-4}, \ldots, h^{-1}$. Следовательно, повторив преобразования применительно к краевьгм условиям (1.8), придем к формулам

$$
\begin{gathered}
L \mathbf{U}-f=-h^{0} f^{1}-\left\{\widetilde{f}-h^{1} L^{2} U^{1}\right\} \\
B^{ \pm} \mathbf{U}-g^{ \pm}=-h^{1} g^{1 \pm}-\left\{\widetilde{g}^{ \pm}-\left[N_{ \pm}^{-1}-1\right]\left(g^{0 \pm}+h^{1} g^{1 \pm}\right)-h^{2} B^{2 \pm} U^{1}\right\}
\end{gathered}
$$

в которых

$$
\begin{array}{cl}
f^{1}=\mathbf{f}+\mathbb{D}_{\zeta} A \mathbb{D}_{y}^{t} U^{1}+\bar{f}^{0}, & g^{1 \pm}=\mathbf{g}^{ \pm} \mp \mathbb{D}_{1} A \mathbb{D}_{y}^{t} U^{1}, \\
\mathbf{f}=\mathbb{D}_{y} A\left(\mathbb{D}_{\zeta}^{t} U^{1}+\mathbb{D}_{y}^{t} U^{0}\right)+\bar{f}^{0}, & \mathbf{g}^{ \pm}=\mathbb{D}_{ \pm} A\left(\mathbb{D}_{\zeta}^{t} U^{1}+\mathbb{D}_{y}^{t} U^{0}\right) .
\end{array}
$$

В силу (1.10) $1-N_{ \pm}(h, y)^{-1}=O\left(h^{2}\right)$, т.е. выражения из фигурных скобок в $(2.26)$ разумно отнести к остатку (малому). Слагаемые $h^{0} f^{1}$ и $h^{1} g^{1 \pm}$ вполне аналогичны составляющим $h^{-1} f^{0}$ и $h^{0} g^{0 \pm}$ в представлениях $(2.5)$ - предъявим к ним подобные (2.6) требования

$$
\int_{\Upsilon(y)} f_{3}^{1}(y, \zeta) d \zeta+g_{3}^{1+}(y)+g_{3}^{1-}(y)=0, \quad y \in \omega
$$

Условия ортогональности (2.28) послужат отправной точкой при обосновании асимптотики в п. 3.4 .

ЗАмечАниЕ 2.3. Соотношения (2.28) можно было получить как условие (2.10), $k=3$, разрешимости задачи вида $(2.9)$ для $U^{2}$. Именно третье условие вьполнялось автоматически (см. (2.22)) и в отличие от первых двух не было востребовано при определении $U^{1}$.

В силу $(2.16),(2.20)$ и (2.27) левая часть (2.28) равна

$$
\int_{\Upsilon(y)} \mathbf{f}_{3} d \zeta+\mathbf{g}_{3}^{+}+\mathbf{g}_{3}^{-}=H \bar{f}_{3}^{0}+\left(e^{3}\right)^{t} \mathbb{D}_{y} \int_{\Upsilon(y)} A\left(\mathbb{D}_{\zeta}^{t} U^{1}+\mathbb{D}_{y}^{t} U^{0}\right) d \zeta .
$$

При обработке последнего слагаемого $I(y)$ используются те же приемы, что и в (2.22): при помоши $(2.4),(2.13)$ с $j=1,(2.21),(2.20)$ и $(2.17),(2.18)$ получаем цепочку равенств

$$
\begin{aligned}
I(y)= & \sum_{i=1}^{2} \frac{\partial}{\partial y_{i}} \int_{\Upsilon(y)}\left(\mathbb{D}_{\zeta}^{t} \zeta e^{i}\right)^{t} A\left(\mathbb{D}_{\zeta}^{t} U^{1}+\mathbb{D}_{y}^{t} U^{0}\right) d \zeta \\
= & -\sum_{i=1}^{2} \frac{\partial}{\partial y_{i}}\left(e^{i}\right)^{t}\left[\int_{\Upsilon(y)} \zeta \mathbb{D}_{\zeta} A\left(\mathbb{D}_{\zeta}^{t} U^{1}+\mathbb{D}_{y}^{t} U^{0}\right) d \zeta\right. \\
& \left.+\sum_{ \pm} \pm\left.\zeta \mathbb{D}_{1} A\left(\mathbb{D}_{\zeta}^{t} U^{1}+\mathbb{D}_{y}^{t} U^{0}\right)\right|_{\zeta= \pm H^{ \pm}}\right] \\
= & \sum_{i=1}^{2} \frac{\partial}{\partial y_{i}}\left[\left(e^{i}\right)^{t} \int_{\Upsilon(y)} \zeta \mathbb{D}_{y} A\left(\mathbb{D}_{\zeta}^{t} U^{0}+\mathbb{D}_{y}^{t} U^{-1}\right) d \zeta\right. \\
& \left.+\left.\sum_{ \pm}\left(e^{i}\right)^{t} \zeta \mathbb{D}_{ \pm} A\left(\mathbb{D}_{\zeta}^{t} U^{0}+\mathbb{D}_{y}^{t} U^{-1}\right)\right|_{\zeta= \pm H_{ \pm}}+\mathscr{F}_{i}^{\prime}\right] \\
= & \sum_{i=1}^{2}\left(e^{i}\right)^{t} \mathbb{D}_{y} \frac{\partial}{\partial y_{i}} \int_{\Upsilon(y)} \zeta A\left(\mathbb{D}_{\zeta}^{t} \mathscr{X}+\mathscr{Y}\right) d \zeta \mathscr{D}\left(\nabla_{y}\right)^{t} w+\sum_{i=1}^{2} \frac{\partial}{\partial y_{i}} \mathscr{F}_{i}^{\prime},
\end{aligned}
$$


где

$$
\begin{aligned}
& \mathscr{F}_{i}^{\prime}(y)=\int_{\Upsilon(y)} \zeta f_{i}^{0}(y, \zeta) d \zeta+\sum_{ \pm} \pm H_{ \pm}(y) g_{i}^{0 \pm}(y), \\
& \mathscr{F}_{3}(y)=H(y) \hat{f}_{3}^{0}(y)+\sum_{i=1}^{2} \frac{\partial}{\partial y_{i}} \mathscr{F}_{i}^{\prime}(y) .
\end{aligned}
$$

Итак, условие (2.28) эквивалентно дифференциальному уравнению

$$
\sum_{i=1}^{2}\left(e^{i}\right)^{t} \mathbb{D}_{y} \frac{\partial}{\partial y_{i}} \int_{\Upsilon(y)} \zeta A \mathscr{Z} d \zeta \mathscr{D}\left(\nabla_{y}\right)^{t} w+\mathscr{F}_{3}=0,
$$

замыкаюшему результирующую систему.

2.5. Результирующая задача. Заметим, что фигурирующие в (2.23) и (2.30) дифференциальные выражения

$$
\mathbb{D}_{y}^{t} e^{1}, \quad \mathbb{D}_{y}^{t} e^{2}, \quad-\mathbb{D}_{y}^{t}\left(e^{1} \partial_{1}+e^{2} \partial_{2}\right) \zeta
$$

оказываются строками матрицы $\mathscr{D}\left(-\nabla_{y}\right) \mathscr{Y}(\zeta)^{t}$. Это наблюдение позволяет записать результируюшую систему одной строкой

$$
\mathscr{D}\left(-\nabla_{y}\right) \mathscr{M}(y) \mathscr{D}\left(\nabla_{y}\right)^{t} w(y)=\mathscr{F}(y), \quad y \in \omega ;
$$

здесь $\mathscr{F}=\left(\mathscr{F}_{1}, \mathscr{F}_{2}, \mathscr{F}_{3}\right)^{t}$ - столбец с компонентами $(2.25),(2.29)$, а $(6 \times 6)$-матрицафункция $\mathscr{M}$ определяется по формуле

$$
\begin{aligned}
\mathscr{M}(y) & =\int_{\Upsilon(y)} \mathscr{Y}(\zeta)^{t} A(y, \zeta)\left(\mathbb{D}_{\zeta}^{t} \mathscr{X}(y, \zeta)+\mathscr{Y}(\zeta)\right) d \zeta \\
& =\int_{\Upsilon(y)}\left(\mathbb{D}_{\zeta}^{t} \mathscr{X}(y, \zeta)+\mathscr{Y}(\zeta)\right)^{t} A(y, \zeta)\left(\mathbb{D}_{\zeta}^{t} \mathscr{X}(y, \zeta)+\mathscr{Y}(\zeta)\right) d \zeta .
\end{aligned}
$$

Последнее представление получается интегрированием по частям при использовании соотношений (2.19) для $\mathscr{X}$.

Так как боковая поверхность пластины жестко защемлена (см. (1.9)), снабдим систему (2.31) краевыми условиями Дирихле

$$
w(y)=0, \quad \partial_{n} w_{3}(y)=0, \quad y \in \partial \omega,
$$

где $n=\left(n_{1}, n_{2}\right)^{t}$ - единичный вектор внешней нормали к контуру $\partial \omega$ и $\partial_{n}=n^{t} \nabla_{y}$ - дифференцирование вдоль $n$.

Теорема 2.4. 1) При всех $y \in \partial \omega$ матрица $\mathscr{M}(y)$ симметрична и положительно определена.

2) Если $\mathscr{F} \in H^{l-1}(\omega)^{2} \times H^{l-2}(\omega)$ при некотором $l \in\{1,2, \ldots\}$, то существует единственное решение $w \in H^{l+1}(\omega)^{2} \times H^{l+2}(\omega)$ задачи (2.31), (2.33) и въполняется оценка

$$
\left\|w ; H^{l+1}(\omega)^{2} \times H^{l+2}(\omega)\right\| \leqslant c\left\|\mathscr{F} ; H^{l-1}(\omega)^{2} \times H^{l-2}(\omega)\right\| .
$$


ДоказАТЕЛЬство. 1) Согласно (2.32) $\mathscr{M}(y)$ - матрища Грама, построенная по столбцам $\mathscr{L}^{1}, \ldots, \mathscr{Z}^{6}$ матрицы $\mathscr{Z}$ из $(2.24)$ при помощи скалярного произведения $(A \cdot, \cdot)_{\Upsilon(y)}$ в $L_{2}(\Upsilon(y))^{6}$. Остается упомянуть, что верхний $(3 \times 3)$-блок матрипы $\mathbb{D}_{\zeta}^{t}$ и нижние $(3 \times 3)$-блоки матрицы $\mathscr{Y ~ н у л е в ы е , ~ т . е . ~ л и н е и ̆ н а я ~ н е з а в и с и м о с т ь ~}$ в $L_{2}(\Upsilon(y))^{6}$ столбцов $\mathscr{Y}^{1}, \ldots, \mathscr{Y}^{6}$ наследуется столбцами $\mathscr{Z}^{1}, \ldots, \mathscr{Z}^{6}$.

2) Нетрудно убедиться в алгебраической комплектности [2] матрицы $\mathscr{D}\left(\nabla_{y}\right)$ из (2.17). Таким образом, оператор системы (2.31) формально положителен [2] и обладает полиномиальньм свойством [39]. Теперь нужное утверждение вытекает из [2; теорема 3.7.6] и [39; теорема 4.2]

Найдем явную формулу для $\mathscr{M}(y)$. С этой целью разобьем матрицы $A, \mathbb{D}_{1}$ и $\mathscr{X}$ на блоки размером $3 \times 3$ :

$$
A=\left(\begin{array}{ll}
A_{(11)} & A_{(12)} \\
A_{(21)} & A_{(22)}
\end{array}\right), \quad \mathbb{D}_{1}=\left(\mathbf{0}_{3}, \mathbb{T}\right), \quad \mathbb{T}=\operatorname{diag}\{\alpha, \alpha, 1\}, \quad \mathscr{X}=\left(\mathscr{X}_{(1)}, \mathscr{X}_{(2)}\right) .
$$

Учитывая строение матрицы $\mathscr{Y}(\zeta)$ из $(2.17)$, положим

$$
\Sigma=A_{(22)} \mathbb{T} \partial_{\zeta} \mathscr{X}+A_{(21)}\left(\mathbf{1}_{3},-\alpha^{-1} \zeta \mathbf{1}_{3}\right) .
$$

Тогда задача (2.19) примет вид

$$
-\partial_{\zeta} \mathbb{T} \Sigma=0 \text { на } \Upsilon(y), \quad \pm \mathbb{T} \Sigma=0 \text { при } \zeta= \pm H_{ \pm}(y) .
$$

Следовательно, $\Sigma(y, \zeta)=0$ и

$$
\mathbb{T} \partial_{\zeta} \mathscr{X}_{(1)}=-A_{(22)}^{-1} A_{(21)}, \quad \mathbb{T} \partial_{\zeta} \mathscr{X}_{(2)}=\alpha^{-1} \zeta A_{(22)}^{-1} A_{(21)} .
$$

Подставляя эти равенства в (2.32), находим, что

$$
\begin{gathered}
\mathscr{M}(y)=\int_{\Upsilon(y)}\left(\begin{array}{cc}
A_{(11)} & A_{(12)} \\
-\alpha^{-1} \zeta A_{(11)} & -\alpha^{-1} \zeta A_{(12)}
\end{array}\right)\left(\begin{array}{cc}
\mathbf{1}_{3} & -\alpha^{-1} \zeta \mathbf{1}_{3} \\
\mathbb{T} \partial_{\zeta} \mathscr{X}_{(1)} & \mathbb{T} \partial_{\zeta} \mathscr{X}_{(2)}
\end{array}\right) d \zeta \\
=\int_{\Upsilon(y)}\left(\begin{array}{cc}
\mathbf{A}(y, \zeta) & -\alpha^{-1} \zeta \mathbf{A}(y, \zeta) \\
-\alpha^{-1} \zeta \mathbf{A}(y, \zeta) & 2 \zeta^{2} \mathbf{A}(y, \zeta)
\end{array}\right) d \zeta \\
\mathbf{A}=A_{(11)}-A_{(12)} A_{(22)}^{-1} A_{(21)} .
\end{gathered}
$$

При этом $\mathbf{A}(y, \zeta)$ - положительно определенная и симметрическая $(3 \times 3)$-матрица. Для пластин постоянной толщины формулы, подобные (2.35), указаны в [30].

Если матрица $A$ кусочно постоянная относительно переменной $\zeta$, т.е. $\Omega_{h}-$ слоистая пластина, то можно выполнить интегрирование в (2.35) и получить явную формулу для матрицы $\mathscr{M}(y)$. Такие формулы в технических теориях известны в случае слоев постоянной толшины (см., например, [28], [30]). Оправдание построенной асимптотики (см. далее замечание 3.7 ) можно истолковать как обоснование упоминавшихся в п. 1.2 классических гипотез Кирхгофа для слабоискривленных слоистых пластин. 


\section{§3. Обоснование асимптотики}

3.1. Вспомогательные неравенства. Доказательство формулируемого неравенства Корна и сопутствующих ему других неравенств откладываем до следующего параграфа (см. пп. 4.2 и 4.4$)$.

TeOpema 3.1. 1) Eсли $u \in \stackrel{\circ}{H}^{1}\left(\Omega_{h}, \Gamma_{h}\right)^{3}, m o$

$$
|u|^{2} \leqslant C E\left(u, u ; \Omega_{h}\right)
$$

причем постоянная $C$ не зависит от и и $h \in(0,1), E$ - функционал из (1.11),

$$
\begin{array}{r}
|u|=\left(\int _ { \Omega _ { h } } \left\{\sum_{i=1}^{2}\left[\left|\nabla_{y} u_{i}\right|^{2}+h^{2} \rho_{h}^{-2}\left(\left|\frac{\partial u_{i}}{\partial z}\right|^{2}+\left|\frac{\partial u_{3}}{\partial y_{i}}\right|^{2}\right)+\rho_{h}^{-2}\left|u_{i}\right|^{2}\right]\right.\right. \\
\left.\left.+\left|\partial_{z} u_{3}\right|^{2}+h^{2} \rho_{h}^{-4}\left|u_{3}\right|^{2}\right\} d x\right)^{1 / 2},
\end{array}
$$

а $\rho_{h}(y)=h+\operatorname{dist}\{y, \partial \omega\}$ - весовая функиия, равная $O(1)$ на удалении от $\Gamma_{h}$ и $O(h)$ в непосредственной близости от $\Gamma_{h}$.

2) Для $u \in H^{1}\left(\Omega_{h}\right)^{3}$ выполняется неравенство

$$
h^{1 / 2} \sum_{i=1}^{2}\left\|\rho_{h}^{-1} u_{i} ; L_{2}\left(\Sigma_{ \pm}\right)\right\|+h^{3 / 2}\left\|\rho_{h}^{-2} u_{3} ; L_{2}\left(\Sigma_{ \pm}\right)\right\| \leqslant c|u|,
$$

в котором обозначения аналогичны использованным выше.

3) Продолжим поле $u \in \stackrel{\circ}{H^{1}}\left(\Omega_{h}, \Gamma_{h}\right)^{3}$ нулем на область (1.1) и введем средние его компонент по переменной $z$ :

$$
\widehat{u}_{j}(y)=h^{-1} H(y)^{-1} \int_{-h H_{-}(y)}^{h H_{+}(y)} u_{j}(y, z) d z .
$$

Справедливо неравенство

$$
\left\|D\left(\nabla_{y}, 0\right)^{t}\left(u-\widehat{u}_{3} e^{3}\right) ; L_{2}\left(\Omega_{h}\right)\right\| \leqslant c E\left(u, u ; \Omega_{h}\right)^{1 / 2}
$$

с постоянной с, не зависящей от и и $h \in(0,1)$.

Далее под $H^{l}\left(\omega \rightarrow H^{s}(\Upsilon)\right)$ понимаем пространство функций в области $\Omega_{1}$ (см. (1.1)), снабженное нормой

$$
\left(\sum_{k=0}^{l} \int_{\omega}\left\|\nabla_{y}^{k} W ; H^{s}(\Upsilon(y))\right\|^{2} d y\right)^{1 / 2}
$$

где $\nabla_{y}^{k} W-$ совокупность производных порядка $k$. 
Лемма 3.2. Пусть $p=0,1,2 u W_{p} \in H^{p+1}\left(\omega \rightarrow L_{2}(\omega)\right)$, причем $W_{1}(y, \zeta)=0$ $u W_{2}(y, \zeta)=\partial_{n} W_{2}(y, \zeta)=0$ при $y \in \partial \omega u \zeta \in \Upsilon(y)$. Тогда верны оценки

$$
\left\|W_{p} ; L_{2}\left(\Omega_{h} \cap \mathbf{V}\right)\right\| \leqslant c h^{p+1}\left\|W_{p} ; H^{p+1}\left(\omega \rightarrow H^{1}(\Upsilon)\right)\right\|,
$$

где $\mathbf{V}=\left\{(y, z): y \in \mathscr{V}_{2 c h}\right\}, \mathscr{V}_{c h}$ - окрестность контура $\partial \omega$, фигурирующая в (1.2), а постоянная с не зависит от $W_{p}$ u $h \in(0,1)$.

ДокАЗАТЕЛЬСтво. Согласно неравенству Пуанкаре-Фридрихса, используюшему условия Дирихле на $\partial \omega$, находим, что при $i=1,2$

$$
\left\|W_{i} ; L_{2}\left(\omega \cap \mathscr{V}_{2 c h} \rightarrow L_{2}(\Upsilon)\right)\right\| \leqslant c h^{i}\left\|\nabla_{y}^{i} W_{i} ; L_{2}\left(\omega \cap \mathscr{V}_{c h} \rightarrow L_{2}(\Upsilon)\right)\right\|
$$

Таким образом, все три неравенства (3.6) сведены к случаю $p=0$. Приступим к проверке самой формулы (3.6), $p=0$. Имеем

$$
\begin{aligned}
& h^{-2} \int_{\mathscr{V} \cap \Omega_{h}}\left|W_{0}\left(y, h^{-1} z\right)\right|^{2} d y d z \\
& \quad \leqslant c h^{-2}\left(h \int_{\mathscr{V}_{2 c h} \cap \omega}\left|\widehat{W}_{0}(y)\right|^{2} d y+\int_{\mathscr{V} \cap \Omega_{h}}\left|W_{0}(y, z)-\widehat{W}_{0}(y)\right|^{2} d y d z\right)
\end{aligned}
$$

$\left(\widehat{W}_{0}\right.$ определено в (3.4)). Благодаря одномерному неравенству Пуанкаре на $\Upsilon(y)$ последний интеграл не превосходит

$$
c h^{2} \int_{\mathscr{V} \cap \Omega_{h}}\left(\left|\nabla_{x} W_{0}\right|^{2}+\left|\nabla_{y} \widehat{W}_{0}(y)\right|^{2}\right) d y d z \leqslant c h^{3}\left\|W_{0} ; H^{1}\left(\omega \rightarrow H^{1}(\Upsilon)\right)\right\|^{2} .
$$

Подчеркнем, что дополнительный множитель $h$ возник из-за замены $z \mapsto \zeta=$ $h^{-1} z$. Рассмотрим первый интеграл справа в (3.7). Сохраним символ $\widehat{W}_{0}$ за функцией $\widehat{W}_{0}$, записанной при помощи естественных криволинейных координат в окрестности $\mathscr{V}_{d}$ контура $\partial \omega$; здесь $t=|n|$ - расстояние до $\partial \omega$ внутри $\omega$, а $s$ - длина дуги на $\partial \omega$. Используя вариант неравенства Харди

$$
\left|\widehat{W}_{0}(0, s)\right|^{2}+\int_{0}^{d} t^{-2}\left|\widehat{W}_{0}(t, s)-\widehat{W}_{0}(0, s)\right|^{2} d t \leqslant c \int_{0}^{d}\left(\left|\partial_{t} \widehat{W}_{0}(t, s)\right|^{2}+\left|\widehat{W}_{0}(t, s)\right|^{2}\right) d t
$$

(см., например, [21; предложение 4.5.2]), обнаруживаем, что

$$
\begin{aligned}
& h^{-1} \int_{\mathscr{V}_{2 c h} \cap \omega}\left|\widehat{W}_{0}(y)\right|^{2} d y \leqslant 2 h^{-1} \int_{\mathscr{V}_{2 c h} \cap \omega}\left(\left|\widehat{W}_{0}(0, s)\right|^{2}+\left|\widehat{W}_{0}(t, s)-\widehat{W}_{0}(0, s)\right|^{2}\right) d y \\
& \quad \leqslant c \int_{\partial \omega} d s\left\{\left|\widehat{W}_{0}(0, s)\right|^{2}+h \int_{0}^{2 c h} t^{2}\left|\widehat{W}_{0}(t, s)-\widehat{W}_{0}(0, s)\right|^{2} d t\right\} \\
& \quad \leqslant c \int_{\partial \omega} d s \int_{0}^{d}\left(\left|\partial_{t} \widehat{W}_{0}(t, s)\right|^{2}+\left|\widehat{W}_{0}(t, s)\right|^{2}\right) d t \leqslant c\left\|W_{0} ; H^{1}\left(\omega \rightarrow L_{2}(\Upsilon)\right)\right\|^{2} .
\end{aligned}
$$

Осталось совместить полученные оценки. 
3.2. О гладкости асимптотических членов. Придадим точный смысл разложениям (2.5). Именно допустим, что

$$
f^{0} \in L_{2}\left(\omega \rightarrow L_{2}(\Upsilon)\right)^{3}, \quad g^{0 \pm} \in L_{2}(\omega)^{3}, \quad \bar{f}_{3}^{0} \in H^{1}(\omega),
$$

где, как обычно, под $H^{-1}(\omega)$ подразумевается пространство функций, имеющих вид

$$
\bar{f}_{3}^{0}(y)=\bar{f}_{3}^{00}(y)-\partial_{1} \bar{f}_{3}^{01}(y)-\partial_{2} \bar{f}_{3}^{02}(y), \quad \bar{f}_{3}^{0 p} \in L_{2}(\omega),
$$

а норма вычисляется по формуле

$$
\left\|\bar{f}_{3}^{0} ; H^{-1}(\omega)\right\|=\inf \left\{\sum_{p=0}^{2}\left\|\bar{f}_{3}^{0 p} ; L_{2}(\omega)\right\|\right\},
$$

причем infimum, берется по всем представлениям (3.9). Сумму норм функций (3.8) в указанных пространствах обозначим $\mathscr{N}_{0}$. Малость остатков в $(2.5)$ обеспечивается предположением: сумма

$$
\begin{aligned}
\widetilde{N}=h^{-1}\left\|\rho_{h}^{2} \widetilde{f}_{3} ; L_{2}\left(\Omega_{h}\right)\right\|+h^{-1 / 2} \sum_{ \pm}\left\|\rho_{h}^{2} \widetilde{g}_{3}^{ \pm} ; L_{2}\left(\Sigma_{ \pm}\right)\right\| \\
+\sum_{i=1}^{2}\left(\left\|\rho_{h} \widetilde{f}_{i} ; L_{2}\left(\Omega_{h}\right)\right\|+h^{1 / 2} \sum_{ \pm}\left\|\rho_{h} \widetilde{g}_{i}^{ \pm} ; L_{2}\left(\Sigma_{ \pm}\right)\right\|\right)
\end{aligned}
$$

есть величина "порядка единицы" . Распределение множителей в (3.10) согласовано с (3.2) и (3.3). Очевидной становится выгода применения весового неравенства Корна (3.1), поскольку соотношение $\mathscr{N}_{0}=O(1)$ сохраняется даже в том случае, если в малой окрестности боковой поверхности пластины $\widetilde{f}$ и $\widetilde{g}^{ \pm}$велики по модулю.

В силу (2.25), (2.29) и (2.34) верна цепочка неравенств

$$
\mathscr{N}_{0} \geqslant C\left\|\mathscr{F} ; L_{2}(\omega)^{2} \times H^{-1}(\omega)\right\| \geqslant c\left\|w ; H^{2}(\omega)^{2} \times H^{3}(\omega)\right\|,
$$

а значит, согласно (2.8), (2.18) и (2.21), (2.12)

$$
\begin{gathered}
U^{k} \in H^{1-k}\left(\omega \rightarrow H^{2}(\Upsilon)\right)^{3}, \quad k=-2,-1,0,1 \\
\left\|U^{k} ; H^{1-k}\left(\omega \rightarrow H^{2}(\Upsilon)\right)\right\| \leqslant c \mathcal{N}_{0} .
\end{gathered}
$$

ЗАмЕчАниЕ 3.3. Члены (3.12) анзаца (2.7) не обладают достаточной гладкостью, и поэтому некоторые из появившихся в $§ 2$ задач следует понимать в обобщенном смысле (как и исходную задачу (1.7)-(1.9)). Мы уклоняемся от очевидных переформулировок и заметим лишш, что в соответствии с (2.27) и (3.9) условие (2.28) также интерпретируется как интегральное тождество

$$
\begin{aligned}
\int_{\omega}\left(\mathbb{D}_{y}^{t} e^{3} v_{3}\right)^{t} \int_{\Upsilon(y)} A\left(\mathbb{D}_{\zeta}^{t} U^{1}+\mathbb{D}_{y}^{t} U^{0}\right) d \zeta d y \\
\quad-\int_{\omega}\left\{\bar{f}_{3}^{00} H v_{3}+\sum_{i=1}^{2} \bar{f}_{3}^{0 i} \partial_{i}\left(H v_{3}\right)\right\} d y=0 \quad \forall v_{3} \in \stackrel{\circ}{H}^{1}(\omega, \partial \omega) .
\end{aligned}
$$

Одно из возможных рассуждений, приводящих к (3.13), реализуется так: функции $f^{0}, g^{0 \pm}$ и $\bar{f}^{0}$ приближаются гладкими, осуществляются все преобразования, указанные в $\S 2$, а затем после подходящего интегрирования по частям делается предельный переход к негладким $f^{0}, g^{0 \pm}$ и $\bar{f}^{0}$. На этом пути получаются все интегральные тождества в данном параграфе, причем их вывод не требует каких-либо оценок, учитьвающих параметр $h$. 
3.3. Приближенное решение. Согласно (3.12) последнее из построенных в $\S 2$ слагаемых $U^{1}$ попадает лишь в $L_{2}\left(\omega \rightarrow H^{2}(\Upsilon)\right)^{3}$ и не может быть включено в асимптотическое приближение из-за недостаточной гладкости по переменным $y$. Для соблюдения условий Дирихле (1.9) умножим предшествующие члены анзаца (2.7) на срезаюшую функцию $X \in C_{0}^{\infty}\left(\omega \backslash \overline{\mathscr{V}_{c h}}\right)$, равную единице при $\operatorname{dist}\{y, \partial \omega\} \geqslant 2 c h$. Эту функцию можно взять зависящей от "быстрой” переменной $h^{-1} t$ (см. доказательство леммы 3.2$)$, т.е.

$$
\left|\nabla_{y}^{k} X(y)\right| \leqslant c_{k} h^{-k}, \quad k=0,1, \ldots
$$

Итак, введем частичную сумму ряда (2.7)

$$
U(x)=h^{-2} U^{-2}(y)+h^{-1} U^{-1}\left(y, h^{-1} z\right)+h^{0} U^{0}\left(y, h^{-1} z\right)
$$

и положим

$$
U_{X}(x)=X(y) U(x) .
$$

По построению и в силу $(1.2),(1.3) U_{X} \in \stackrel{\circ}{H^{1}}\left(\Omega_{h}, \Gamma_{h}\right)^{3}$.

Лемма 3.4. Вектор-функции (3.15) и (3.16) связаньи неравенством

$$
\left|U-U_{X}\right|+\left\|\varepsilon(U)-\varepsilon\left(U_{X}\right) ; L_{2}\left(\Omega_{h}\right)\right\| \leqslant c h^{0} \mathscr{N}_{0} .
$$

ДоКАЗАТЕЛЬСТво. Носитель разности $U-U_{X}=(1-X) U$ расположен в окрестности $\mathbf{V}$, фигурируюшей в лемме 3.2 , на которую нам предстоит ссылаться. Благодаря краевым условиям (2.23), структуре начальных членов (2.8) и соотношениям (3.12) компоненты векторов

$$
\begin{aligned}
U^{-2}, \partial_{z} U^{-2} & \in H^{3}\left(\omega \rightarrow H^{1}(\Upsilon)\right), \\
U^{-1}, \partial_{z} U^{-1}, \nabla_{y} U^{-2} & \in H^{2}\left(\omega \rightarrow H^{1}(\Upsilon)\right), \\
U^{0}, \partial_{z} U^{0}, \nabla_{y} U^{-1} & \in H^{1}\left(\omega \rightarrow H^{1}(\Upsilon)\right)
\end{aligned}
$$

можно в лемме 3.2 принять за $W_{2}, W_{1}, W_{0}$ соответственно (градиент $\nabla_{y} U^{0}$ обрабатывается отдельно). Заметим еще, что в интеграле (3.2), суженном на $\Omega_{h} \cap \mathbf{V}$, множители $\rho_{h}^{-m}$ безболезненно заменяются на $h^{-m}$. Выполняя дифференцирование при учете (3.14), находим, что левая часть (3.17) не превосходит

$$
\begin{aligned}
c \sum_{p=0}^{2} h^{-p}\left\{h^{-1}\left\|U^{-p} ; L_{2}\left(\Omega_{h} \cap \mathbf{V}\right)\right\|+\left\|\nabla_{y} U^{-p} ; L_{2}\left(\Omega_{h} \cap \mathbf{V}\right)\right\|\right. \\
\left.\quad+\left\|\partial_{z} U^{-p} ; L_{2}\left(\Omega_{h} \cap \mathbf{V}\right)\right\|\right\} \\
\leqslant c \sum_{p=0}^{2} h^{-p}\left\{h^{-1} h^{p+1}\left\|U^{-p} ; H^{p+1}\left(\omega \rightarrow H^{1}(\Upsilon)\right)\right\|\right. \\
\quad+h^{(p-1)+1}\left\|\nabla_{y} U^{-p} ; H^{p}\left(\omega \rightarrow H^{1}(\Upsilon)\right)\right\| \\
\left.\quad+h^{p+1}\left\|h^{-1} \partial_{\zeta} U^{-p} ; H^{p+1}\left(\omega \rightarrow H^{1}(\Upsilon)\right)\right\|\right\} \\
\leqslant c \sum_{p=0}^{2}\left\|U^{-p} ; H^{p+1}\left(\omega \rightarrow H^{2}(\Upsilon)\right)\right\| \leqslant c \mathscr{N}_{0} .
\end{aligned}
$$


При первом оценивании использовались неравенства (3.6), а также очевидная формула

$$
\left\|\nabla_{y} U^{0} ; L_{2}\left(\Omega_{h} \cap \mathbf{V}\right)\right\| \leqslant\left\|\nabla_{y} U^{0} ; L_{2}\left(\Omega_{h}\right)\right\| .
$$

Дальнейшие вькладки опирались на (3.12). Лемма доказана.

3.4. Вычисление и оценивание невязок. Аналогично п. 2.4 имеем

$$
\begin{aligned}
L U_{X}- & f=L(1-X) U+h^{-4} L^{0} U^{-2} \\
& +h^{-3}\left(L^{0} U^{-1}+L^{1} U^{-2}\right)+h^{-2}\left(L^{0} U^{0}+L^{1} U^{-1}+L^{2} U^{-2}\right) \\
& +h^{-1}\left(L^{1} U^{0}+L^{2} U^{-1}-f^{0}\right)+h^{0}\left(L^{2} U^{0}-\bar{f}^{0}\right)-\widetilde{f} \\
= & L(1-X) U-h^{-1} L^{0} U^{1}+h^{0}\left(L^{2} U^{0}-\bar{f}^{0}\right)-\widetilde{f} \\
B^{ \pm} U_{X} & -g^{ \pm}=B^{ \pm}(1-X) U+h^{-3} N_{ \pm}^{-1} B^{0 \pm} U^{-2} \\
& +h^{-2} N_{ \pm}^{-1}\left(B^{0 \pm} U^{-1}+B^{1 \pm} U^{-2}\right) \\
& +h^{-1} N_{ \pm}^{-1}\left(B^{0 \pm} U^{0}+B^{1 \pm} U^{-1}+B^{2 \pm} U^{-2}\right) \\
& +h^{0} N_{ \pm}^{-1}\left(B^{1 \pm} U^{-2}+B^{2 \pm} U^{-1}-g^{0 \pm}\right) \\
& +h^{1} B^{2 \pm} U^{0}+\left(N_{ \pm}^{-1}-1\right) g^{0 \pm}-\widetilde{g}^{ \pm} .
\end{aligned}
$$

Здесь применялись соотношения (2.13) с $j=-2,-1,0$, а слагаемые $-L^{0} U^{1}$ и $-B^{0 \pm} U^{1}$ появились в соответствии с $(2.21)$ и $(2.13), j=1$.

Положим $R=U_{X}-u$ и заметим, что $L U_{X}-f=L R, B^{ \pm} U_{X}-g^{ \pm}=B^{ \pm} R$, а сам вектор $R$ удовлетворяет краевым условиям (1.9). Умножим (3.18) скалярно на $R$ и проинтегрируем по частям при учете краевых условий (3.19). В результате получим:

$$
\begin{aligned}
2 E\left(R, R ; \Omega_{h}\right)= & \left\langle A D^{t}(1-X) U, D^{t} R\right\rangle-\left\langle A \mathbb{D}_{\zeta}^{t} U^{1}, \mathbb{D}_{z}^{t} R\right\rangle \\
& +\left\{\left\langle A \mathbb{D}_{y}^{t} U^{0}, \mathbb{D}_{y}^{t} R\right\rangle-h\left(\bar{f}_{3}^{00}, H \widehat{R}_{3}\right)_{\omega}-h \sum_{i=1}^{2}\left(\bar{f}_{3}^{0 i}, \partial_{i} H \widehat{R}_{3}\right)_{\omega}\right\} \\
& +\left\{\langle\widetilde{f}, R\rangle+\sum_{ \pm}\left[\left(\widetilde{g}^{ \pm}, R\right)_{\Sigma_{ \pm}}+\left(\left(N_{ \pm}^{-1}-1\right) g^{0 \pm}, R\right)_{\Sigma_{ \pm}}\right]\right\} \\
\equiv & I_{X}-I_{1}+I_{0}+\widetilde{I} .
\end{aligned}
$$

Скалярное произведение в $L_{2}\left(\Omega_{h}\right)$ обозначено через $\langle\cdot, \cdot\rangle$. Кроме того, $D=D\left(\nabla_{x}\right)$ и $\mathbb{D}_{z}=h^{-1} \mathbb{D}_{\zeta}$; в выражении $\left\langle f^{0}, R\right\rangle$ учтены условия $(2.6),(3.9)$ и произведено интегрирование по $z$, т.е. $\widehat{R}_{3}$ определено согласно (3.4). Помимо формулы Грина (1.11) использовались формулы, получающиеся из нее заменой операторов $L, B^{ \pm}$на $L^{0}, N_{ \pm}^{-1} B^{0 \pm}$ или $L^{2}, N_{ \pm}^{-1} B^{2 \pm}$.

Оценим слагаемые $I_{\ldots}$ в в (3.20). В силу леммы 3.4

$$
\left|I_{X}\right| \leqslant c\left\|\varepsilon(U)-\varepsilon\left(U_{X}\right) ; L_{2}\left(\Omega_{h}\right)\right\| \times\left\|\varepsilon(R) ; L_{2}\left(\Omega_{h}\right)\right\| \leqslant C \mathscr{N}_{0} E\left(R, R ; \Omega_{h}\right)^{1 / 2} .
$$

Следуюший член $I_{1}$ обрабатывается так:

$$
\begin{aligned}
\left|I_{1}+I_{10}\right| & =\left|\left\langle A \mathbb{D}_{\zeta}^{t} U^{1},\left(\mathbb{D}_{z}^{t}+\mathbb{D}_{y}^{t}\right) R\right\rangle\right| \leqslant c\left\|\mathbb{D}_{\zeta}^{t} U^{1} ; L_{2}\left(\Omega_{h}\right)\right\| \times\left\|D^{t} R ; L_{2}\left(\Omega_{h}\right)\right\| \\
& \leqslant c h^{1 / 2}\left\|\mathbb{D}_{\zeta}^{t} U^{1} ; L_{2}\left(\omega \rightarrow L_{2}(\Upsilon)\right)\right\| \times\left\|\varepsilon(R) ; L_{2}\left(\Omega_{h}\right)\right\| \\
& \leqslant c h^{1 / 2} \mathcal{N}_{0} E\left(R, R ; \Omega_{h}\right)^{1 / 2}, \\
I_{10} & =\left\langle A \mathbb{D}_{\zeta}^{t} U^{1}, \mathbb{D}_{y}^{t} R\right\rangle .
\end{aligned}
$$


Оставшийся интеграл $I_{10}$ присоединим к $I_{0}$. Кроме того, в интегральном тождестве (3.13) положим $v_{3}=\widehat{R}_{3}$ и вычтем левую часть из $I_{0}+I_{10}$. В итоге получаем

$$
I_{0}+I_{10}=\left\langle A\left(\mathbb{D}_{y}^{t} U^{0}+\mathbb{D}_{\zeta}^{t} U^{1}\right), \mathbb{D}_{y}^{t}\left(R-e^{3} \widehat{R}_{3}\right)\right\rangle,
$$

а значит, по теореме $3.1(3)$

$$
\begin{aligned}
\left|I_{0}+I_{10}\right| \leqslant & c h^{1 / 2}\left(\left\|\mathbb{D}_{y}^{t} U^{0} ; L_{2}\left(\omega \rightarrow L_{2}(\Upsilon)\right)\right\|\right. \\
& \left.+\left\|\mathbb{D}_{\zeta}^{t} U^{1} ; L_{2}\left(\omega \rightarrow L_{2}(\Upsilon)\right)\right\|\right) E\left(R, R ; \Omega_{h}\right)^{1 / 2} \\
\leqslant & c h^{1 / 2} \mathscr{N}_{0} E\left(R, R ; \Omega_{h}\right)^{1 / 2}
\end{aligned}
$$

Здесь, как и в (3.22), были применены соотношения (3.12), а множитель $h^{1 / 2}$ появился из-за замены $z \mapsto \zeta=h^{-1} z$. Наконец, согласно теореме $3.1(1,2)$ и формулам (3.10), (3.8), (1.10) выполняются неравенства

$$
|\widetilde{I}| \leqslant c\left(\widetilde{N}|R|+\mathscr{N}_{0} h^{2} \sum_{ \pm}\left\|R ; L_{2}\left(\Sigma_{ \pm}\right)\right\|\right) \leqslant c\left(\widetilde{\mathscr{N}}+h^{1 / 2} \mathscr{N}_{0}\right) E\left(R, R ; \Omega_{h}\right)^{1 / 2}
$$

3.5. Теорема об асимптотике. Собирая оценки (3.21)-(3.24) и основьваясь на (3.20), обнаруживаем, что

$$
E\left(R, R ; \Omega_{h}\right)^{1 / 2} \leqslant c\left(\mathscr{N}_{0}+\widetilde{\mathscr{N}}\right) .
$$

Отсюда и из неравенств $(1.12),(3.1)$ и (3.17) вьводим основной результат статьи.

ТЕОРема 3.5. Пусть правъе части $f$ и $g$ подчиненъ требованиям (2.5), (2.6) и (3.8), (3.10), определяющим, в частности, величинь $\mathscr{N}_{0}$ и $\widetilde{N}$. Тогда решение и задачи (1.7)-(1.9) и асимптотическое приближение (3.15), построенное при помощи формул (2.8), (2.18) по решению ш результирующей задачи (2.31), (2.33) с правой частью (2.25), (2.29), связаны неравенством

$$
|u-U|+\left\|\varepsilon(u)-\varepsilon(U) ; L_{2}\left(\Omega_{h}\right)\right\| \leqslant c\left(\mathscr{N}_{0}+\widetilde{\mathscr{N}}\right),
$$

в котором постоянная с зависит от $\omega, H_{ \pm} u A$, но не от $h \in(0,1]$ и функций $f^{0}, g^{0 \pm}, \bar{f}^{0}$ и $\widetilde{f}, \widetilde{g}^{ \pm}$, составляющих правье части исходной задачи.

Приведем упрощенные асимптотические формулы для смещений и деформаций (напряжений), вытекаюшие из (3.25).

СлЕДСтвиЕ 3.6. В условиях теоремы 3.5 верны оценки

$$
\begin{aligned}
\left\|\rho_{h}^{-2}\left(u_{3}-h^{2} w_{3}\right) ; L_{2}\left(\Omega_{h}\right)\right\| & \leqslant c h^{-1}\left(\mathscr{N}_{0}+\widetilde{\mathcal{N}}\right), \\
\left\|\rho_{h}^{-1}\left(u_{i}-h^{-1}\left(w_{i}-\zeta \frac{\partial w_{3}}{\partial y_{i}}\right)\right) ; L_{2}\left(\Omega_{h}\right)\right\| & \leqslant c\left(\mathscr{N}_{0}+\widetilde{\mathscr{N}}\right), \\
\left\|\varepsilon(u)-h^{-1}\left(\mathbb{D}_{\zeta}^{t} \mathscr{X}+\mathscr{Y}\right) \mathscr{D}\left(\nabla_{y}\right)^{t} w ; L_{2}\left(\Omega_{h}\right)\right\| & \leqslant c\left(\mathscr{N}_{0}+\widetilde{\mathscr{N}}\right) .
\end{aligned}
$$


ДокАЗАТЕЛЬСТво. В соответствии с (1.4), (2.1) и (2.8), (2.14), (2.17), (2.18) вьполняются равенства

$$
\begin{aligned}
\varepsilon(U)= & \left(h^{-1} \mathbb{D}_{\zeta}^{t}+\mathbb{D}_{y}^{t}\right) U=h^{-3} \mathbb{D}_{\zeta}^{t} U^{-2}+h^{-2}\left(\mathbb{D}_{\zeta}^{t} U^{-1}+\mathbb{D}_{y}^{t} U^{-2}\right) \\
& +h^{-1}\left(\mathbb{D}_{\zeta}^{t} U^{0}+\mathbb{D}_{y}^{t} U^{-1}\right)+h^{0} \mathbb{D}_{y}^{t} U^{0} \\
= & h^{-1}\left(\mathbb{D}_{\zeta}^{t} \mathscr{X}+\mathscr{Y}\right) \mathscr{D}\left(\nabla_{y}\right)^{t} w+\mathbb{D}_{y}^{t} U^{0} .
\end{aligned}
$$

Так как согласно (3.12)

$$
\left\|\mathbb{D}_{y}^{t} U^{0} ; L_{2}\left(\Omega_{h}\right)\right\| \leqslant c h^{1 / 2}\left\|U^{0} ; H^{1}\left(\omega \rightarrow L_{2}(\Upsilon)\right)\right\| \leqslant c h^{1 / 2} \mathscr{N}_{0},
$$

последняя оценка в (3.26) выводится из (3.25) и (3.27). Для того чтобы проверить первые две оценки, достаточно убедиться в справедливости формулы $\left|U^{0}\right|_{0} \leqslant c h^{0} \mathscr{N}_{0}$, где $|u|_{0}^{2}$ - фрагмент интеграла из (3.2), включающий смещения $u_{i}$ и $u_{3}$, но не их производные. Поскольку $h^{2} \rho_{h}(y)^{-2} \leqslant c$, нужная формула получается так:

$$
\begin{aligned}
\left|U^{0}\right|_{0}^{2} & \leqslant c h^{2}\left\|\rho_{h}^{-1} U^{0} ; L_{2}\left(\Omega_{h}\right)\right\|^{2} \leqslant c h^{3}\left\|\rho_{h}^{-1} U^{0} ; L_{2}\left(\omega \rightarrow L_{2}(\Upsilon)\right)\right\|^{2} \\
& \leqslant c h\left\|U^{0} ; H^{1}\left(\omega \rightarrow H^{1}(\Upsilon)\right)\right\|^{2} \leqslant c h \mathscr{N}_{0}^{2} .
\end{aligned}
$$

В пояснении нуждается предпоследний переход. Если $\omega^{\prime}-$ собственная подобласть $\omega$ и $\rho_{h}(y) \geqslant c>0$ при $y \in \omega^{\prime}$, то

$$
\left\|\rho_{h}^{-1} U^{0} ; L_{2}\left(\omega^{\prime} \rightarrow L_{2}(\Upsilon)\right)\right\| \leqslant c\left\|U^{0} ; L_{2}\left(\omega^{\prime} \rightarrow L_{2}(\Upsilon)\right)\right\| \leqslant c \mathscr{N}_{0} .
$$

Поэтому, не ограничивая общности, будем считать, что при $\operatorname{dist}\{y, \partial \omega\} \geqslant d$ $U^{0}(y, \zeta)=0$. Далее следуем схеме проверки оценки (3.6), $p=0$, из леммы 3.4, но применяем другой вариант неравенства Харди

$$
\begin{aligned}
& h \int_{0}^{d}(h+n)^{-2}|\mathscr{Z}(n)|^{2} d n=-2 h \int_{0}^{d}(h+n)^{-2} \int_{n}^{d} \mathscr{Z}(\tau) \partial_{\tau} \mathscr{Z}(\tau) d \tau d n \\
& \quad=-2 h \int_{0}^{d} \mathscr{Z}(\tau) \partial_{\tau} \mathscr{Z}(\tau) \int_{0}^{\tau}(h+n)^{-2} d n d \tau \leqslant 2 \int_{0}^{d} \frac{\tau}{h+\tau}\left|\mathscr{Z}(\tau) \partial_{\tau} \mathscr{Z}(\tau)\right| d \tau \\
& \quad \leqslant 2 d\left(\int_{0}^{d}\left|\partial_{\tau} \mathscr{Z}(\tau)\right|^{2} d \tau\right)^{1 / 2}\left(\int_{0}^{d}(h+\tau)^{-2}|\mathscr{Z}(\tau)|^{2} d \tau\right)^{1 / 2}
\end{aligned}
$$

справедливый для $\mathscr{Z} \in H^{1}(0, d)$ в случае $\mathscr{Z}(d)=0$.

Слагаемое $U^{0}$ безболезненно удаляется из асимптотического решения (3.15) только при рассмотрении самих смещений - оно принимает участие в формировании асимптотик деформаций (3.27) и напряжений $\sigma(U)$. Оценки $(3.25)$ и $(3.26)$ в самом деле служат оправданием построенных асимптотических представлений, поскольку нормы отделенных асимптотических членов

$|U|, \quad\left\|\varepsilon(U) ; L_{2}\left(\Omega_{h}\right)\right\|, \quad\left|h^{-2} U^{-2}+h^{-1} U^{-1}\right|_{0}, \quad\left\|h^{-1}\left(\mathbb{D}_{\zeta}^{t} \mathscr{X}+\mathscr{Y}\right) \mathscr{D}^{t} w ; L_{2}\left(\Omega_{h}\right)\right\|$ суть $O\left(h^{1 / 2} \mathscr{N}_{0}\right)$. 
Полная оценка (3.25) является асимптотически точной: как установлено в [17], [40] для цилиндрической изотропной пластины, энергетическая норма (левая часть $(3.25))$ главного члена пограничного слоя, возникающего вблизи боковой поверхности пластины, но не учитываемого в асимптотическом решении (3.15), как раз и составляет $O(1)$. Пограничный слой служит для компенсации невязки, оставленной приближенным решением в условиях Дирихле (1.9). В промежуточном приближении (3.16) эту роль исполняла срезающая функция $X$, причем вносимая ею погрешность имела тот же порядок $h^{0}$ (см. (3.17)).

Требования гладкости (3.8) нельзя ослабить без потери точности. Так, при наличии у функции $f^{0}$ сингулярностей, выводящих ее из класса $L_{2}\left(\omega \rightarrow L_{2}(\Upsilon)\right)^{3}$, показатель степени $h$ в мажоранте из (3.25) становится отрицательным - это связано с явлением внутренних пограничных слоев, обладающих трехмерной структурой (см. [41]-[43] и др.).

Замечание 3.7. Предположение о гладкости матрицы $A$ в законе Гука (1.6) можно ослабить и перейти к рассмотрению слоистых пластин, у которых $A-$ кусочно гладкая матрица-функция с разрывами на поверхностях

$$
\Sigma_{n}=\left\{x: y \in \omega, z=h H_{n}(y)\right\}, \quad n=1, \ldots, N
$$

Здесь $H_{n}$ - гладкие функции, $-H_{-}<H_{1}<\cdots<H_{N}<H_{+}$. Теперь задача (1.7)-(1.9) дополняется условиями сопряжения

$$
\begin{gathered}
D(\nu(y)) A\left(y, H_{n}(y)-0\right) D\left(\nabla_{x}\right)^{t} u\left(y, h H_{n}(y)-0\right) \\
=D(\nu(y)) A\left(y, H_{n}(y)+0\right) D\left(\nabla_{x}\right)^{t} u\left(y, h H_{n}(y)+0\right), \\
u\left(y, h H_{n}(y)-0\right)=u\left(y, h H_{n}(y)+0\right), \quad y \in \omega, \quad n=1, \ldots, N,
\end{gathered}
$$

в которых фигурируют односторонние пределы при $\zeta \rightarrow H_{n}(y) \pm 0$, а $\nu(y)$ - нормаль к $\Sigma_{n}$. Впрочем, условия сопряжения (3.29) исчезают при формулировке задачи (1.7)-(1.9) как интегрального тождества. При выводе результирующей системы (2.31) можно ограничиться такими, обобщенными, постановками предельных задач (2.13) (ср. с замечанием 3.3), т.е. финальные формулы (2.32) и (2.35) сохраняются и для слоистых пластин. Остается отметить, что при обосновании асимптотики мы также обращались к подобию (3.20) интегрального тождества.

3.6. Пологие оболочки. Один из наиболее часто встречающихся типов слабо изогнутых пластин - пологие оболочки, геометрия которых описывается такой формулой:

$$
\Omega_{h}=\{x: \tau \in \widetilde{\omega},|\sigma|<h / 2\} .
$$

Здесь $\widetilde{\omega}$ - область с гладкой границей $\partial \widetilde{\omega}$, расположенная на слабоискривленной гладкой поверхности $\widetilde{\Sigma}($ ее кривизны суть $O(h)), \tau-$ система координат на $\widetilde{\Sigma}, \sigma-$ поперечная координата, т.е. $|\sigma|=\operatorname{dist}\{x, \widetilde{\Sigma}\}$. Простейшим примером служит пологая сферическая оболочка, заключенная между сферами

$$
\mathbb{S}_{ \pm}=\left\{x:|y|^{2}+|z+l|^{2}=\left(\sqrt{l^{2}+r_{0}^{2}} \pm \frac{1}{2} h\right)^{2}\right\}
$$


и проецируюшаяся на круг $\omega=\left\{y:|y|<r_{0}\right\}$ с фиксированным радиусом $r_{0}$. Центр $(0,-l)$ сфер удален от плоскости $\omega$, т.е. $l=l(h)$, а произведение $l_{0}=h l$ не зависит от $h$. Переходя к обозначениям из (1.1), получаем уравнения для величин $H_{ \pm}(h, y)$ :

$$
|y|^{2}+h^{2} H_{ \pm}^{2}+2 l_{0} H_{ \pm}=r_{0}^{2} \pm \sqrt{l_{0}^{2}+h^{2} r_{0}^{2}}+\frac{1}{4} h^{2},
$$

из которого выводим, что

$$
\begin{gathered}
H_{ \pm}(h, y)=H_{ \pm}^{0}(y)+h^{2} \widetilde{H}_{ \pm}(h, y), \quad H_{ \pm}^{0}(y)=\frac{1}{2}\left( \pm 1+l_{0}^{-1}\left(r_{0}^{2}-|y|^{2}\right)\right) \\
\left|\nabla_{y}^{k} \widetilde{H}_{ \pm}(h, y)\right| \leqslant c_{k}, \quad k=0,1, \ldots
\end{gathered}
$$

Дополнительная зависимость функций $H_{ \pm}$от параметра $h$ не привносит каких-либо осложнений. Поскольку в механике малый параметр - некая условность, более правильньм оказывается повторение процедуры из $§ 2$ при сохранении зависимостей $H_{ \pm}$от $h$. В этом случае завершающий этап - асимптотический анализ результирующей задачи, содержащей параметр $h,-$ весьма прост. Также физически осмысленной является перезапись всех уравнений в криволинейных координатах, привязанных к срединной поверхности оболочки

$$
\widetilde{\Sigma}=\left\{x: z=h H_{0}(h, y)\right\}
$$

где $H_{0} \sim 2^{-1}\left(H_{+}-H_{-}\right)$. Компоненты единичного вектора $\mathbf{n}=\left(\mathbf{n}_{1}, \mathbf{n}_{2}, \mathbf{n}_{3}\right)^{t}$ нормали к $\widetilde{\Sigma}$ имеют вид

$$
\begin{gathered}
\mathbf{n}_{3}=N_{0}(h, y)^{-1}, \quad \mathbf{n}_{i}=-h N_{i}(h, y) N_{0}(h, y)^{-1}, \quad i=1,2, \\
N_{i}(h, y)=\partial_{i} H_{0}(h, y), \quad N_{0}(h, y)=\left[1+h^{2}\left(N_{1}(h, y)^{2}+N_{2}(h, y)^{2}\right)\right]^{1 / 2}
\end{gathered}
$$

(cp. с (1.10)). Два перпендикулярных вектора $\mathbf{s}^{i}=\left(\mathbf{s}_{1}^{i}, \mathbf{s}_{2}^{i}, \mathbf{s}_{3}^{i}\right)^{t}$ в плоскости, касательной к $\widetilde{\Sigma}$, можно определить так:

$$
\begin{aligned}
& \mathbf{s}_{3}^{i}=h N_{i}(h, y) N_{0}(h, y)^{-1}, \\
& \mathbf{s}_{i}^{i}=\left\{1-h^{2}\left[N_{0}(h, y)-1\right]^{-1} N_{3-i}(h, y)^{2}\right\} N_{0}(h, y)^{-1}, \\
& \mathbf{s}_{2}^{1}=\mathbf{s}_{1}^{2}=h^{2}\left[N_{0}(h, y)-1\right]^{-1} N_{1}(h, y) N_{2}(h, y) N_{0}(h, y)^{-1} .
\end{aligned}
$$

Теперь переход к криволинейным координатам в анзаце (2.7) и дифференциальном операторе из результирующей системы (2.31) не составляет труда. Наконец, схема обоснования асимптотики остается прежней, так как все производные функций $y \mapsto H_{ \pm}(h, y)$ равномерно ограничены при $h \in(0,1)$ и $y \in \bar{\omega}$.

3.7. Замечания о пограничных слоях. Если пластина цилиндрическая и $H_{ \pm}$- постоянные, то предельная задача, описывающая явление пограничного слоя, получается в результате введения двух быстрых переменных $\eta_{1}=h^{-1} t$ и $\eta_{2}=h^{-1} z$, где $(n, s)$ - естественные криволинейные координаты в окрестности контура $\partial \omega, s$ - длина дуги на $\partial \omega$ и $-n=t$ - расстояние до $\partial \omega$ вдоль внутренней нормали. Подчеркнем, что для переменной $s$ сохраняется начальный масштаб и 
она оказывается параметром в двумерной задаче анизотропной теории упругости в полосе $\Pi=\mathbb{R}_{+} \times\left(-H_{-}, H_{+}\right)$. Необходимую информацию о разрешимости такой задачи можно почерпнуть из $[21 ; \S 5.7]$ и [44], а полное описание процедуры приведено, например, в [11]-[19] и [20; гл. 15, 16].

Для пластин переменной толщины схема построения пограничного слоя в целом остается прежней. Незначительные изменения связаны с необходимостью спрямить границы, что делается при помощи замены

$$
\begin{gathered}
(t, z) \mapsto \eta=\left(\eta_{1}, \eta_{2}\right) \\
\eta_{1}=h^{-1} t, \quad \eta_{2}=H(t, s)^{-1}\left[H(0, s) h^{-1} z+H_{+}(0, s) H_{-}(t, s)-H_{-}(0, s) H_{+}(t, s)\right]
\end{gathered}
$$

(см. [34]). После выделения главных частей дифференциальных операторов относительно параметра $h$ (их коэффициенты требуется разложить в ряды Маклорена по переменной $t$ ) осушествляется формальный переход к $h=0$ и получается задача теории упругости в полуполосе $\Pi(s)=\mathbb{R}_{+} \times\left(-H_{-}(0, s), H_{+}(0, s)\right)$, зависящей от $s \in \partial \omega$. По сравнению с возможностью пользоваться "простыми” быстрыми переменными $\left(h^{-1} t, h^{-1} z\right)$ и обрабатьвать задачи в областях со слабо возмушенными границами введение координат (3.30) обладает значительными преимуществами, продемонстрированньми на многочисленных примерах в [20].

Новые моменты обнаруживаются при рассмотрении пластин с острыми краями. Предположим, что условие $H>0$, введенное в п. 1.1, выполняется в $\omega$, но нарушается всюду на $\partial \omega$, причем для простоты

$$
H_{ \pm}(t, s)=t^{\gamma} \mathscr{H}_{ \pm}(s), \quad \gamma>0, \quad \mathscr{H}_{ \pm} \in C^{\infty}(\partial \omega), \quad \mathscr{H}=\mathscr{H}_{+}+\mathscr{H}_{-}>0
$$

В этом случае пластина оканчивается ребром и в соответствии с (2.35) результирующая система (2.31) вырождается на $\partial \omega$. Как известно (см. [33], [45] и др.), количество краевых условий, необходимых для корректной постановки задачи Дирихле, варьируется в зависимости от порядка вырождения. Так, при $\gamma \in(0,1 / 3)$ нужны все четыре условия (2.33), но при $\gamma \in[1 / 3,2 / 3)$ и $\gamma \in[2 / 3,1)$ следует ограничиться соответственно наборами краевых условий

$$
w_{1}(y)=w_{2}(y)=0, \quad w_{3}(y)=0, \quad y \in \partial \omega
$$

и

$$
w_{1}(y)=w_{2}(y)=0, \quad y \in \partial \omega .
$$

В случае $\gamma \geqslant 1$ краевые условия не требуются вовсе. Эти эффекты, разумеется, тесно связаны с явлением пограничного слоя. Дело в том, что формула (3.30) теряет смысл в ситуации (3.31) и полуполоса не выступает в качестве предельной области. Согласно [46], [34] и [20; $\S 12.4]$ правильной оказывается замена $(y, z) \mapsto\left(s, \xi_{1}, \xi_{2}\right)$,

$$
\xi_{1}=h^{\alpha} t, \quad \xi_{2}=h^{\alpha} z, \quad \alpha=(1-\gamma)^{-1}, \quad \gamma \in(0,1)
$$

которая в окрестности контура $\{x: y \in \partial \omega, z=0\}$ преобразует $\Omega_{h}$ в $\{(s, \xi)$ : $s \in \partial \omega, \xi \in \Pi(s)\}$, где П $(s)$ - параболическое множество:

$$
\Pi(s)=\left\{\xi \in \mathbb{R}^{2}: \xi_{1}>0, \xi_{1}^{-\gamma} \xi_{2} \in\left(-\mathscr{H}_{-}(s), \mathscr{H}_{+}(s)\right)\right\} .
$$


Весовые неравенства Корна в областях такого типа изучались в [47], [48], а асимптотические разложения решений - в [35], [36]. Упомянутые результаты показывают, что при $\gamma \in(0,1 / 3)$ имеются четыре условия затухания решения задачи в $(s)$, а при $\gamma \in(1 / 3,2 / 3)$ и $\gamma \in(2 / 3,1)$ их становится соответственно три и два (ровно столько же краевых условий указано в (3.32) и (3.33)). В случаях $\gamma=1 / 3$ и $\gamma=2 / 3$ приходится оперировать с ограниченными решениями. Подчеркнем, что при $\gamma=1$ или $\gamma>1$ (на краю пластины ребро или нулевое заострение) замена (3.34) неправомочна, поскольку $\alpha=\infty$ или $\alpha<0$. Это означает отсутствие пограничного слоя (cp. с [34]) и согласуется с упоминавшимся исчезновением краевых условий.

Построение асимптотических разложений для пластин с острым краем усложняется в значительной степени: появляются нецелые показатели степеней $h$ и из-за особенностей производных решений приходится обращаться либо к методу сращиваемых разложений ([49], [50] и др.), либо к процедуре перераспределения невязок [20]. Сколь-нибудь подробных публикаций на эту тему автор не знает.

\section{§4. Весовое неравенство Корна}

4.1. План доказательства теоремы 3.1. Неравенство (3.1) в случае цилиндрической пластины $\omega \times(-h / 2, h / 2)$ проверено в [26], причем отправным пунктом послужило аналогичное неравенство без весовых множителей (т.е. $\rho_{h}=1$ в (3.2)), установленное в [6]. Переход к весовой норме (3.2) был достигнут в [26] при помощи удобного разбиения $\left\{\omega_{j}: j=1, \ldots, J_{h}\right\}$ области $\omega$ и обработки интеграла из $(3.2)$, суженного на цилиндр $\omega_{j} \times(-h / 2, h / 2)$. Нечто подобное предстоит сделать и в этом параграфе. Заметим, что благодаря условиям (1.2) и (1.3) поле

$u \in \stackrel{\circ}{H}{ }^{1}\left(\Omega_{h}, \Gamma_{h}\right)^{3}$ продолжается нулем на пластину $\Omega_{h}^{0}$ из $(1.1)$ с боковой поверхностью $\Gamma_{h}^{0}=\left\{x: y \in \partial \omega,-h H_{-}(y)<z<h H_{+}(y)\right\}$. Более того, считаем, что функции $H_{ \pm}$продолжены с сохранением основных свойств на область $\omega^{\prime}$, содержащую $\bar{\omega}$, и вводим множества $\Omega_{h}^{\prime}, \Gamma_{h}^{\prime}$ и т.п. Далее оперируем с полями $u$ из $\stackrel{\circ}{H^{1}}\left(\Omega_{h}^{0}, \Gamma_{h}^{0}\right)$ или $\stackrel{\circ}{H^{1}}\left(\Omega_{h}^{\prime}, \Gamma_{h}^{\prime}\right)$.

Пусть $\left\{\omega^{1}, \ldots, \omega^{N}\right\}$ - фиксированное (не зависящее от $h$ ) покрытие области $\omega$. Если оно достаточно мелкое, то окажется выполненным такое условие: при каждом $n=1, \ldots, N$ существуют число $\varkappa_{n}>0$ (фиксированное) и декартова система координат $\left(y^{n}, z^{n}\right)$ (зависяшая от $\left.h\right)$, для которых

$$
G_{h}^{n}=\left\{x: y^{n} \in g^{n},\left|z^{n}\right| \leqslant h \varkappa_{n}\right\} \subset \Omega_{h}^{\prime} .
$$

При этом $g^{n}-$ множество в плоскости $z^{n}=0$, проекции которого на плоскость $z=0$ совпадают с $\omega_{n} ;$ справедливы формулы

$$
\begin{gathered}
\left(y, z-z_{0}^{n}(h)\right)^{t}=\Theta(h)\left(y^{n}, z^{n}\right)^{t} \\
\left|z_{0}^{n}(h)\right| \leqslant c h, \quad\left|\Theta(h)-\mathbf{1}_{3}\right| \leqslant c h
\end{gathered}
$$

где $\Theta(h)$ и $\mathbf{1}_{3}-$ унитарная и единичная матрицы размером $3 \times 3$. Приведем пояснения. В силу (4.1) вовнутрь $\Omega_{h}^{\prime}$ помещается цилиндрическая пластина $G_{h}^{n}$ толщиной $2 h \varkappa_{n}$, срединное сечение которой проецируется на $\omega_{n}$. Соотношения (4.2) показывают, что переход к системе координат, привязанной к $G_{h}^{n}$, осуществляется сдвигом вдоль оси $z$ на расстояние $O(h)$ и поворотом осей на малые углы. 
Вывод весового неравенства Корна для пластины переменной толщины осуществляется по следуюшей схеме. Сначала отбираются области $\omega^{1}, \ldots, \omega^{K}$, пересекаюшиеся с контуром $\partial \omega$. Для "прямых" пластин $G_{h}^{1}, \ldots, G_{h}^{K}$ применяются весовые неравенства Корна вида (3.1) из [26], справедливость которых обеспечивается условиями Дирихле на $\Gamma_{h}^{0}$. Затем эти неравенства распространяются на "искривленные" пластины $\Omega_{h}^{n}=\left\{x \in \Omega_{h}^{\prime}: y \in \omega^{n}\right\}, n=1, \ldots, K$ (см. далее леммы 4.2 и 4.3). На этом завершается первый этап и получается весовое неравенство (3.1), суженное на фиксированную окрестность боковой поверхности пластины $\Omega_{h}$. На втором этапе рассматриваются области $\omega^{K+1}, \ldots, \omega^{L}$, имеющие непустые пересечения с хотя бы одним из множеств $\omega^{1}, \ldots, \omega^{K}$. Так как $\rho_{h}(y)>c>0$ при $y \in \omega^{K+1} \cup \cdots \cup \omega^{L}$, неравенства $(3.1)$ на $G_{h}^{K+1}, \ldots, G_{h}^{L}$ перестают быть весовыми по сушеству и устанавливаются при помоши результатов [6] (см. далее лемму 4.1(2)). Как и ранее, леммы 4.2 и 4.3 позволяют заменить $G_{h}^{n}$ на $\Omega_{h}^{n}$, $n=K+1, \ldots, L$, и еше более отодвинуться от края пластины $\Omega_{h}$. Очередной этап повторяет предыдуший, и требуется конечное их число для перебора всех элементов покрытия $\left\{\omega^{n}: n=1, \ldots, N\right\}$.

Описанная схема реализуется в конце параграфа, а начинаем мы с проверки более простых утверждений 2) и 3) теоремы 3.1, вытекающих из (3.1).

4.2. Теорема о следах. Следуюшее тождество очевидно:

$$
\begin{aligned}
\mid u_{j}(y, & \left.H_{+}(y)\right)\left.\right|^{2}+\left|u_{i}\left(y,-H_{-}(y)\right)\right|^{2} \\
& =\int_{-h H_{-}(y)}^{h H_{+}(y)}(h H(y))^{-1} \frac{\partial}{\partial z}\left[\left(2 z-h\left(H_{+}(y)-H_{-}(y)\right)\right) u_{j}(y, z)^{2}\right] d z .
\end{aligned}
$$

Вьполнив дифференцирование, оцениваем последний интеграл величиной

$$
\frac{c}{h} \int_{-h H_{-}(y)}^{h H_{+}(y)}\left(h^{2}\left|\partial_{z} u_{j}(y, z)\right|^{2}+\left|u_{j}(y, z)\right|^{2}\right) d z .
$$

При $j=1,2$ умножим полученное неравенство на $\rho_{h}(y)^{-2}$, а при $j=3$ - на $h^{2} \rho_{h}(y)^{-4}$. Для вьвода (3.3) осталось проинтегрировать по $y \in \omega$ и заметить, что $h^{4} \rho_{h}^{-4}\left|\partial_{z} u_{3}\right|^{2} \leqslant\left|\partial_{z} u_{3}\right|^{2}$.

4.3. Неравенство для $u-e^{3} \widehat{u}_{3}$. Разобьем окрестность замыкания $\bar{\omega}$ на квадраты $\left\{y:\left|y_{i}-h k_{i}\right|<h / 2, k_{i} \in\{0, \pm 1, \ldots\}, i=1,2\right\}$. Пусть $q$ - один из таких квадратов с центром в точке $y^{q}$, имеющий непустое пересечение с $\omega$ и потому лежащий в $\omega^{\prime}$. Введем еще призму $Q=\left\{(y, z): y \in q,-h H_{-}(y)<z<h H_{+}(y)\right\}$ с криволинейными основаниями и поместим в нее шар $B$ с радиусом $h r$ и центром на прямой $y=y^{q}$. Так как $H_{ \pm}-$непрерывные функции, число $r>0$ можно взять не зависящим от $h$ и положения квадрата $q$.

Для сужения поля $u \in \stackrel{\circ}{H^{1}}\left(\Omega_{h}^{\prime}, \Gamma_{h}^{\prime}\right)^{3}$ на $Q$ справедливо представление

$$
u(x)=u^{\perp}(x)+u^{0}(x),
$$

в котором $u^{0}(x)=d(x) a, a-$ столбец в $\mathbb{R}^{6}$,

$$
d(x)=\left(\begin{array}{cccccc}
1 & 0 & 0 & 0 & \alpha z & -\alpha y_{2} \\
0 & 1 & 0 & -\alpha z & 0 & \alpha y_{1} \\
0 & 0 & 1 & \alpha y_{2} & -\alpha y_{1} & 0
\end{array}\right)
$$


(ср. с матрицей $D(y, z)$, у которой для явной аналогии переставлены столбцы). Так как $u^{0}$ - смещение $Q$ как жесткого целого, верны равенства

$$
\varepsilon(u)=\varepsilon\left(u^{\perp}\right), \quad E(u, u ; Q)=E\left(u^{\perp}, u^{\perp} ; Q\right),
$$

которые вытекают, например, из формул $(1.4),(1.11)$ и соотношения $D\left(\nabla_{x}\right)^{t} d(x)=$ $\mathbf{0}_{6}$ (см. (1.6) и (4.5)). Положим

$$
a=d_{B}^{-1} \int_{B} d(x)^{t} u(x) d x, \quad d_{B}=\int_{B} d(x)^{t} d(x) d x
$$

и подчеркнем, что $d_{B}$ - матрица Грама размером $6 \times 6$, положительно определенная и симметрическая. Не ограничивая общности, можно считать, что начало координат помешено в центр шара $B$. Тогда простые вычисления показывают, что

$$
d_{B}=\frac{4}{3} \pi r^{3} h^{3}\left(\begin{array}{cc}
\mathbf{1}_{3} & \mathbf{0}_{3} \\
\mathbf{0}_{3} & p \mathbf{1}_{3}
\end{array}\right), \quad p=\frac{1}{5} r^{2} h^{2} .
$$

Заметим, что в силу (4.4) и (4.7)

$$
\int_{Q} d(x)^{t} u^{\perp}(x) d x=\int_{Q} d(x)^{t} u(x) d x-\int_{Q} d(x)^{t} d(x) d x a=d_{B} a-d_{B} a=0 .
$$

Благодаря условиям ортогональности справедливо такое неравенство Корна:

$$
E\left(u^{\perp}, u^{\perp} ; Q\right) \geqslant c\left(\left\|\nabla_{x} u^{\perp} ; L_{2}(Q)\right\|^{2}+h^{-2}\left\|u^{\perp} ; L_{2}(Q)\right\|^{2}\right)
$$

(см. [3], [4] и др.). Обращаем внимание на два обстоятельства. Во-первых, множитель $h^{-2}$ появился в связи с заменой $x \mapsto \xi=h^{-1} x$, исключающей мальй параметр $h$ и переводящей $Q$ в “единичную” призму

$Q_{1}=\left\{\xi=\left(\xi^{\prime}, \xi_{3}\right) \in \mathbb{R}^{3}:\left|\xi_{i}\right|<1 / 2, i=1,2 ;-H_{-}\left(y^{q}+h \xi^{\prime}\right)<\xi_{3}<H_{+}\left(y^{q}+h \xi^{\prime}\right)\right\}$.

Во-вторых, множество $Q$ оказывается звездным относительно шара $B$, и поэтому согласно [4] постоянная $c>0$ в (4.9) выражается через отношение диаметров $(2 h r)^{-1} \operatorname{diam} Q=(2 r)^{-1} \operatorname{diam} Q_{1}$, не зависит от $h$ и ее можно взять общей для всех квадратов из разбиения $\{q\}$.

В силу (3.4) и (4.10) имеем

$$
\begin{gathered}
\left\|\nabla_{y}\left(u^{\perp}-e^{3} \widehat{u}_{3}^{\perp}\right) ; L_{2}(Q)\right\|^{2} \leqslant 2\left(\left\|\nabla_{y} u^{\perp} ; L_{2}(Q)\right\|^{2}+\left\|\nabla_{y} \widehat{u}_{3}^{\perp} ; L_{2}(Q)\right\|^{2}\right) \\
\leqslant c\left(E\left(u^{\perp}, u^{\perp} ; Q\right)+\int_{q}\left\{h \sum_{ \pm}\left|u_{3}^{\perp}\left(y, \pm h H_{ \pm}(y)\right)\right|^{2}\right.\right. \\
\left.\left.\quad+\int_{-h H_{-}(y)}^{h H_{+}(y)}\left(\left|\nabla_{y} u_{3}^{\perp}(y, z)\right|^{2}+\left|u_{3}^{\perp}(y, z)\right|^{2}\right) d z\right\} d y\right) .
\end{gathered}
$$

Тождество (4.3) позволяет оценить сумму по \pm из фигурных скобок посредством интеграла из тех же скобок. Таким образом, ссылаясь еще раз на (4.10), находим, что

$$
\left\|D\left(\nabla_{y}, 0\right)^{t}\left(u^{\perp}-e^{3} \widehat{u}_{3}^{\perp}\right) ; L_{2}(Q)\right\|^{2} \leqslant c E\left(u^{\perp}, u^{\perp} ; Q\right) .
$$


Пусть $d_{3}$ - третья строка матрицы (4.5). Непосредственными вычислениями убеждаемся в справедливости равенств

$$
\begin{gathered}
d(y, z)-e^{3} \widehat{d}_{3}(y)=\left(\begin{array}{cccccc}
1 & 0 & 0 & 0 & \alpha z & -\alpha y_{2} \\
0 & 1 & 0 & -\alpha z & 0 & \alpha y_{1} \\
0 & 0 & 0 & 0 & 0 & 0
\end{array}\right) \\
D\left(\nabla_{y}, 0\right)^{t}\left(d(y, z)-e^{3} \widehat{d}_{3}(y)\right)=\mathbf{0}_{6} .
\end{gathered}
$$

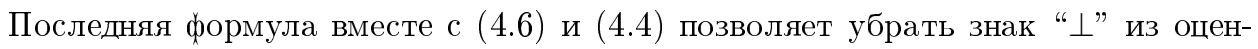
ки (4.11) и получить, что

$$
\left\|D\left(\nabla_{y}, 0\right)^{t}\left(u-e^{3} \widehat{u}_{3}\right) ; L_{2}\left(Q_{h}\right)\right\|^{2} \leqslant c E(u, u ; Q)
$$

Осталось просуммировать последнее неравенство по всем элементам разбиения пластины $\Omega_{h}$ на призмы $Q$.

4.4. Доказательство теоремы 3.1 (неравенство Корна). Сначала получим вспомогательные неравенства на цилиндрических пластинах $G_{h}^{n}$, введенных в п. 4.1. Вектор $v \in H^{1}\left(G_{h}^{n}\right)^{3}$ записываем в координатах $\left(y^{n}, z^{n}\right)$, и его проекции обозначаем через $v_{1}^{n}, v_{2}^{n}$ и $v_{3}^{n}$. Кроме того,

$$
\begin{aligned}
\left|v ; G_{h}^{n}\right|_{1}^{2}= & \int_{G_{h}^{n}}\left\{\sum_{i=1}^{2}\left(\left|\nabla_{y^{n}} v_{i}^{n}\right|^{2}+h^{2}\left(\left|\frac{\partial v_{i}^{n}}{\partial z^{n}}\right|^{2}+\left|\frac{\partial v_{3}^{n}}{\partial y_{i}^{n}}\right|^{2}\right)+\left|v_{i}^{n}\right|^{2}\right)\right. \\
& \left.+\left|\frac{\partial v_{3}^{n}}{\partial z^{n}}\right|^{2}+h^{2}\left|v_{3}^{n}\right|^{2}\right\} d y^{n} d z^{n} .
\end{aligned}
$$

Если дополнительно $v=0$ на $T_{h}^{n}=\left\{\left(y^{n}, z^{n}\right): y^{n} \in t^{n},\left|z^{n}\right|<h \varkappa_{n}\right\}$, где $t^{n}-$ непустая открытая дуга на контуре $\partial g^{n}$ (граница срединного сечения $G_{h}^{n}$ ), то под $\left|v ; G_{h}^{n}\right|_{\rho}^{2}$ понимается интеграл (4.12), в котором по аналогии с (3.2) распределены степени весового множителя $\rho_{h}^{n}\left(y^{n}\right)=h+\operatorname{dist}\left\{y^{n}, t^{n}\right\}$.

Лемма 4.1. 1) Для $v \in \stackrel{\circ}{H^{1}}\left(G_{h}^{n}, T_{h}^{n}\right)^{3}$ выполняется неравенство

$$
\left|v ; G_{h}^{n}\right|_{\rho}^{2} \leqslant c E\left(v, v ; G_{h}^{n}\right)
$$

2) Если $v \in H^{1}\left(G_{h}^{n}\right)^{3}$ и $r^{n}$ - несобственная подобласть $g^{n}, R_{h}^{n}=\left\{\left(y^{n}, z^{n}\right)\right.$ : $\left.y^{n} \in r^{n},\left|z^{n}\right|<h \varkappa_{n}\right\}, m o$

$$
\left|v ; G_{h}^{n}\right|_{1}^{2} \leqslant c\left(E\left(v, v ; G_{n}^{h}\right)+\left|v ; R_{h}^{n}\right|_{1}^{2}\right) .
$$

Постоянные с в (4.13) и (4.14) не зависят от $v$ и $h \in(0,1)$. 
ДоказАТЕльство. Утверждение 1) установлено в [26]. Проверим неравенство (4.14), используя приемы из [6]. После замен

$$
z^{n} \mapsto \zeta^{n}=h^{-1} z^{n}, \quad v_{3}^{n} \mapsto V_{3}^{n}=h v_{3}^{n}
$$

получаем, что при $h \in(0,1]$

$$
\begin{aligned}
\int_{G_{h}^{n}}\{ & \left.\sum_{i=1}^{2}\left(\left|\frac{\partial v_{i}^{n}}{\partial y_{i}^{n}}\right|^{2}+\left|\frac{\partial v_{i}^{n}}{\partial z^{n}}+\frac{\partial v_{3}^{n}}{\partial y_{i}^{n}}\right|^{2}\right)+\left|\frac{\partial v_{1}^{n}}{\partial y_{2}^{n}}+\frac{\partial v_{2}^{n}}{\partial y_{1}^{n}}\right|^{2}+\left|\frac{\partial v_{3}^{n}}{\partial z^{n}}\right|^{2}\right\} d y^{n} d z^{n} \\
& +h \int_{G_{h}^{n}}\left\{\sum_{i=1}^{2}\left(\left|\frac{\partial v_{i}^{n}}{\partial y_{i}^{n}}\right|^{2}+h^{-2}\left|\frac{\partial v_{i}^{n}}{\partial \zeta^{n}}+\frac{\partial V_{3}^{n}}{\partial y_{i}^{n}}\right|^{2}\right)\right. \\
& \left.+\left|\frac{\partial v_{1}^{n}}{\partial y_{2}^{n}}+\frac{\partial v_{2}^{n}}{\partial y_{1}^{n}}\right|^{2}+h^{-4}\left|\frac{\partial V_{3}^{n}}{\partial \zeta^{n}}\right|^{2}\right\} d y^{n} d \zeta^{n} \\
\geqslant & h \int_{G_{h}^{n}}\left\{\sum_{i=1}^{2}\left(\left|\frac{\partial v_{i}^{n}}{\partial y_{i}^{n}}\right|^{2}+\left|\frac{\partial v_{i}^{n}}{\partial \zeta^{n}}+\frac{\partial V_{3}^{n}}{\partial y_{i}^{n}}\right|^{2}\right)\right. \\
& \left.+\left|\frac{\partial v_{1}^{n}}{\partial y_{2}^{n}}+\frac{\partial v_{2}^{n}}{\partial y_{1}^{n}}\right|^{2}+\left|\frac{\partial V_{3}^{n}}{\partial \zeta^{n}}\right|^{2}\right\} d y^{n} d \zeta^{n}
\end{aligned}
$$

В силу (1.12) и (1.5) левая часть (4.16) оценивается сверху величиной $c E\left(v, v ; G_{h}^{n}\right)$. Интеграл $I$ из правой части лишш несущественными в данном контексте множителями отличается от упругой энергии поля $\left(v_{1}^{n}, v_{2}^{n}, V_{3}^{n}\right)^{t}$ в теле $G_{1}^{n}$ с единичными размерами. Неравенство Корна на $G_{1}^{n}$ (см. [2]-[4] и др.) может быть записано так:

$$
\begin{aligned}
h \sum_{i=1}^{2}\left\|v_{i}^{n} ; H^{1}\left(G_{1}^{n}\right)\right\|^{2}+h\left\|V_{3}^{n} ; H^{1}\left(G_{1}^{n}\right)\right\|^{2} & \\
& \leqslant c\left(h I+h \sum_{i=1}^{2}\left\|v_{i}^{n} ; L_{2}\left(R_{1}^{n}\right)\right\|^{2}+h\left\|V_{3}^{n} ; L_{2}\left(R_{1}^{n}\right)\right\|^{2}\right) .
\end{aligned}
$$

Разумеется, постоянная $c$ в (4.17) не зависит от $v$ и $h$. Теперь нужно сделать замены, обратные для (4.15). Правая часть полученного неравенства не превосходит правой части (4.14). Его левая часть отличается от интеграла (4.12) лишш тем, что при $\left|\partial_{z^{n}} v_{3}^{n}\right|^{2}$ появился множитель $h^{4}$. Этот множитель удаляется без последствий, так как сам член $\left|\partial_{z^{n}} v_{3}^{n}\right|^{2}$ можно найти в первом подынтегральном выражении из (4.16).

Очередное утверждение позволит перейти от норм, возникших в лемме 4.1, к норме (3.2) на $G_{h}^{n}$, т.е. осушествить замену координат (4.2). Пусть $\omega^{n} \subset \omega^{\prime}-$ краевой элемент покрытия $\left\{\omega^{n}\right\}$ области $\omega$, т.е. $\varnothing \neq \omega^{n} \cap \partial \omega \subset \tau^{n}$, а множества $g^{n}$ и $t^{n}$, фигурирующие в лемме 4.1 , проецируются на $\omega^{n}$ и $\tau^{n}$. Очевидно, что весовые множители $\rho_{h}(y)$ и $\rho_{h}^{n}\left(y^{n}\right)$ оказываются эквивалентными и о них в дальнейшем заботиться не нужно. Для внутреннего элемента $\overline{\omega^{n}} \subset \omega$ под $\left|v ; G_{h}^{n}\right|_{\rho}^{2}$ будет подразумеваться интеграл $(4.12)$, но эквивалентность весов $\rho_{h}(y)$ и $\rho_{h}^{n}\left(y^{n}\right)=1$ на $G_{h}^{n}$ сохранится. 
ЛЕмма 4.2. Пусть и-векторное поле $v$, указанное в лемме 4.1 (1) или 2)), но записанное в координатах $(y, z)$, привязанных $к$ пластине $\Omega_{h}$. Пусть еще $|u|_{G, n}^{2}-$ интеграл из $(3.2)$, суженный $c \Omega_{h}$ на $G_{h}^{n}$. Тогда неравенства

$$
|u|_{G, n}^{2} \leqslant c\left|v ; G_{h}^{n}\right|_{\rho}^{2} \leqslant C|u|_{G, n}^{2}
$$

выполняются с постоянными, не зависящими от и и $h \leqslant 1$.

ДокаЗАТЕЛЬство. Сначала рассмотрим фрагмент левой части (4.18), свободный от производных компонент $u_{j}$ :

$$
I_{0}=\int_{G_{h}^{n}}\left\{\rho_{h}^{-2}\left|u_{1}\right|^{2}+\rho_{h}^{-2}\left|u_{2}\right|^{2}+h^{2} \rho_{h}^{-4}\left|u_{3}\right|^{2}\right\} d y d z
$$

(ср. с (3.2)). Поскольку столбцы $u$ и $v$ связаны формулой $u=\Theta(h) v$, в соответствии с (4.2) заключаем, что

$$
\begin{aligned}
I_{0} \leqslant & c \int_{G_{h}^{n}}\left\{\rho_{h}^{-2}\left(\left|v_{1}\right|^{2}+h^{2}\left|v_{2}\right|^{2}+h^{2}\left|v_{3}\right|^{2}\right)+\rho_{h}^{-2}\left(h^{2}\left|v_{1}\right|^{2}+\left|v_{2}\right|^{2}+h^{2}\left|v_{3}\right|^{2}\right)\right. \\
& \left.+h^{2} \rho_{h}^{-4}\left(h^{2}\left|v_{1}\right|^{2}+h^{2}\left|v_{2}\right|^{2}+\left|v_{3}\right|^{2}\right)\right\} d y^{n} d z^{n} .
\end{aligned}
$$

По определению весовой функции $h^{-1}>\rho_{h}(y)^{-1}>c_{\rho}>0$ в $\omega^{\prime}$, а значит, $h^{2} \rho_{h}^{-2}\left|v_{3}\right|^{2} \leqslant c_{\rho}^{-2} h^{2} \rho_{h}^{-4}\left|v_{3}\right|^{2}$ и $h^{4} \rho_{h}^{-4}\left|v_{i}\right|^{2} \leqslant \rho_{h}^{-2}\left|v_{i}\right|^{2}, i=1,2$. Отсюда следует, что $I_{0} \leqslant c\left|v ; G_{h}^{n}\right|_{\rho}^{2}$.

Обработаем оставшуюся часть $I_{1}$ выражения $|u|_{G, n}^{2}$. Согласно (4.2) для присутствующих в ней производных справедливы неравенства

$$
\begin{aligned}
& \left|\frac{\partial u_{k}}{\partial y_{i}}(y, z)\right|^{2} \leqslant c\left(\left|\frac{\partial v_{k}}{\partial y_{i}^{n}}\left(y^{n}, z^{n}\right)\right|^{2}+h^{2}\left|\nabla_{\left(y^{n}, z^{n}\right)} v\left(y^{n}, z^{n}\right)\right|^{2}\right), \\
& \left|\frac{\partial u_{k}}{\partial z}(y, z)\right|^{2} \leqslant c\left(\left|\frac{\partial v_{k}}{\partial z^{n}}\left(y^{n}, z^{n}\right)\right|^{2}+h^{2}\left|\nabla_{\left(y^{n}, z^{n}\right)} v\left(y^{n}, z^{n}\right)\right|^{2}\right),
\end{aligned}
$$

где $k=1,2,3$ и $i=1,2$. Следовательно, оценивание $I_{1}$ можно произвести так: для членов, имеющих весовой множитель $h^{2} \rho_{h}^{-2}$, воспользоваться соотношениями $h^{4} \rho_{h}^{-2} \leqslant h^{2} \leqslant 1$ и $h^{4} \rho_{h}^{-2} \leqslant h^{2} \rho_{h}^{-2}$, а для членов без множителя - соотношениями $h^{2} \leqslant 1$ и $h^{2} \leqslant c_{\rho}^{-2} h^{2} \rho_{h}^{-2}$.

Второе неравенство в (4.18) получится при помощи точно таких же рассуждений, если заметить, что матрица $\Theta(h)^{-1}$, осуществляющая обратные замены, по-прежнему удовлетворяет неравенству $\left|\Theta(h)^{-1}-\mathbf{1}_{3}\right| \leqslant c h$.

Последняя из лемм предоставляет возможность распространить неравенство Корна с $G_{h}^{n}$ на $\widetilde{\Omega}_{h}^{n}=\left\{x \in \Omega_{h}^{n}: y \in \omega^{n}, \operatorname{dist}\left\{y, \partial \omega^{n}\right\}>\sqrt{2} h\right\}$. Сужение основания искривленной пластины происходит по чисто техническим причинам - используется разбиение $\{q\}$ окрестности множества $\bar{\omega}$, введенное в п. 4.3. Разумеется, можно было бы подобрать разбиение, приспособленное к форме $\omega^{n}$, и тем самьм заменить $\widetilde{\Omega}_{h}^{n}$ на $\Omega_{h}^{n}=\left\{x \in \Omega_{h}^{\prime}: y \in \omega^{n}\right\}$, однако отрезание пограничных полосок шириной $O(h)$ не нарушает намеченную процедуру вывода неравенства (3.1), поскольку покрытие $\left\{\omega^{n}\right\}$ не зависит от $h$ (глубина перехлеста его элементов достаточна) и поле $u \in \stackrel{\circ}{H^{1}}\left(\Omega_{h}, \Gamma_{h}\right)^{3}$ продолжено нулем на увеличенную пластину $\Omega_{h}^{\prime}$ (около края $\Gamma_{h}$ образован запас). Далее через $|u|_{\Omega, n}^{2},|u|_{Q}^{2}$ или $|u|_{B}^{2}$ обозначается интеграл из $(3.2)$, суженный на области $\widetilde{\Omega}_{h}^{n}, Q$ или $B$ соответственно. 
Лемма 4.3. Для $u \in \stackrel{\circ}{H^{1}}\left(\Omega_{h}, \Gamma_{h}\right)^{3} \subset \stackrel{\circ}{H^{1}}\left(\Omega_{h}^{\prime}, \Gamma_{h}^{\prime}\right)^{3}$ вылолняется соотношение

$$
|u|_{\Omega, n}^{2} \leqslant c\left(|u|_{G, n}^{2}+E\left(u, u ; \widetilde{\Omega}_{h}^{n}\right)\right)
$$

причем постоянная с не зависит от и $и$ $h \in(0,1)$.

ДоКАЗАТЕЛЬСТво. Пусть $q$ - один из квадратов со стороной $h$, содержащийся в $\omega^{n}$. Как и в п. 4.3 , определим призму $Q$ с криволинейными основаниями, но выбор шара $B$ подчиним дополнительному условию $B \subset Q \cap G_{h}^{n}$ (при этом остальные требования соблюдаются). Так как $\rho_{h}(y)^{-2} \leqslant h^{-2}$ в норме (3.2), из (4.6) и (4.10) вытекает, что

$$
\left|u^{\perp}\right|_{Q}^{2} \leqslant c E\left(u^{\perp}, u^{\perp} ; Q\right)=c E(u, u ; Q) .
$$

Далее будет проверена такая формула для составляющей $u^{0}(x)=d(x) a$ в (4.6):

$$
\left|u^{0}\right|_{Q}^{2} \leqslant c|u|_{B}^{2} .
$$

Постоянные в (4.20) и (4.21) можно взять общими для всех квадратов, попадаюших в $\omega^{n}$. Поэтому, суммируя эти неравенства, приходим к искомому соотношению (4.19):

$$
\begin{aligned}
|u|_{\Omega, n}^{2} & =\sum_{\ldots}|u|_{Q}^{2} \leqslant 2\left(\sum_{\ldots}\left|u^{0}\right|_{Q}^{2}+\sum_{\ldots}\left|u^{\perp}\right|_{Q}^{2}\right) \\
& \leqslant 2 c\left(\sum_{\ldots}|u|_{B}^{2}+\sum_{\ldots} E(u, u ; Q)\right) \leqslant 2 c\left(|u|_{G, n}^{2}+E\left(u, u ; \widetilde{\Omega}_{h}^{n}\right)\right) .
\end{aligned}
$$

Итак, осталось удостовериться в справедливости (4.21). Сделаем это, совместив центр $\left(y^{B}, z^{B}\right)$ шара $B$ с началом координат (чтобы не писать всюду $d\left(y-y^{B}\right.$, $z-z^{B}$ ) вместо $\left.d(x)\right)$. Согласно (4.7)

$$
a \equiv\left(a_{1}, \ldots, a_{6}\right)^{t}=d_{B}^{-1} J, \quad J \equiv\left(J_{1}, \ldots, J_{6}\right)^{t}=\int_{B} d(x)^{t} u(x) d x .
$$

Поэтому прямое вычисление нормы $|d a|_{B}$ при учете явного выражения (4.8) для матрицы $d_{B}$ показывает, что

$$
\begin{aligned}
|d a|_{B}^{2} \leqslant & c h^{3}\left\{\rho^{-2}\left(\left|a_{1}\right|^{2}+\left|a_{2}\right|^{2}\right)+h^{2} \rho^{-4}\left|a_{3}\right|^{2}+h^{2} \rho^{-2}\left(\left|a_{4}\right|^{2}+\left|a_{5}\right|^{2}\right)+\left|a_{6}\right|^{2}\right\} \\
\leqslant & C h^{-3}\left\{\rho^{-2}\left(\left|J_{1}\right|^{2}+\left|J_{2}\right|^{2}\right)+h^{2} \rho^{-4}\left|J_{3}\right|^{2}\right. \\
& \left.+h^{-2} \rho^{-2}\left(\left|J_{4}\right|^{2}+\left|J_{5}\right|^{2}\right)+h^{-4}\left|J_{6}\right|^{2}\right\}
\end{aligned}
$$

где $\rho=\rho_{h}\left(y^{q}\right)$ и при некоторых постоянных $C, c>0$

$$
c \rho \leqslant \rho_{h}(y) \leqslant C \rho .
$$

При $i=1,2$ благодаря (4.5) и (4.23) имеем

$$
\left|J_{i}\right|^{2}=\left|\int_{B} u_{i} d x\right|^{2} \leqslant c \operatorname{mes}_{3} B \int_{B}\left|u_{i}\right|^{2} d x \leqslant c h^{3} \rho^{2} \int_{B} \rho_{h}^{-2}\left|u_{i}\right|^{2} d x \leqslant c h^{3} \rho^{2}|u|_{B}^{2} .
$$


Аналогично

$$
\left|J_{3}\right|^{2} \leqslant c h^{3} \int_{B}\left|u_{3}\right|^{2} d x \leqslant c h \rho^{4}|u|_{B}^{2} .
$$

Положим $P(x)=\left|y-y^{B}\right|^{2}+\left|z-z^{B}\right|^{2}-r^{2} h^{2}$, где $r h-$ радиус шара $B$. Тогда

$$
\begin{aligned}
\left|J_{6-i}\right|^{2} & =\frac{1}{2}\left|\int_{B}\left(z u_{i}-y_{i} u_{3}\right) d x\right|^{2}=\frac{1}{8}\left|\int_{B}\left(u_{i} \frac{\partial P}{\partial z}-u_{3} \frac{\partial P}{\partial y_{i}}\right) d x\right|^{2} \\
& =\frac{1}{8}\left|\int_{B} P\left(\frac{\partial u_{i}}{\partial z}-\frac{\partial u_{3}}{\partial y_{i}}\right) d x\right|^{2} \leqslant c h^{3}\left(\max _{B} P\right)^{2} \int_{B}\left(\left|\frac{\partial u_{i}}{\partial z}\right|^{2}+\left|\frac{\partial u_{3}}{\partial y_{i}}\right|^{2}\right) d x \\
& \leqslant c h^{5} \rho^{2} \int_{B} h^{2} \rho_{h}^{-2}\left(\left|\frac{\partial u_{i}}{\partial z}\right|^{2}+\left|\frac{\partial u_{3}}{\partial y_{i}}\right|^{2}\right) d x \leqslant c h^{5} \rho^{2}|u|_{B}^{2} .
\end{aligned}
$$

Наконец,

$$
\begin{aligned}
\left|J_{6}\right|^{2} & =\frac{1}{2}\left|\int_{B}\left(x_{1} u_{2}-x_{2} u_{1}\right) d x\right|^{2}=\frac{1}{8}\left|\int_{B} P\left(\frac{\partial u_{1}}{\partial x_{2}}-\frac{\partial u_{2}}{\partial x_{1}}\right) d x\right|^{2} \\
& \leqslant c^{7} \int_{B}\left(\left|\frac{\partial u_{1}}{\partial x_{2}}\right|^{2}+\left|\frac{\partial u_{2}}{\partial x_{1}}\right|^{2}\right) d x \leqslant \operatorname{ch}^{7}|u|_{B}^{2} .
\end{aligned}
$$

Подставляя в (4.22) полученные оценки для $\left|J_{1}\right|, \ldots,\left|J_{6}\right|$, приходим к (4.19), что и требовалось.

В заключение обсудим порядок применения доказанных лемм. Если область $\omega^{1}$ пересекается с $\partial \omega$, то при учете продолжения поля $u$ нулем на $\Omega_{h}^{\prime}$ обращаемся последовательно к леммам 4.1(1), 4.2 и 4.3. Они доставляют неравенство Корна (3.1) на $\widetilde{\Omega}_{h}^{1}$. Пусть теперь площадь пересечения $\omega^{K+1}$ и $\omega^{1}$ ненулевая. Тогда к неравенству Корна (3.1) на $\omega^{1} \cup \omega^{K+1}$ приводят леммы 4.1(2), 4.2 и 4.3. Все ситуации, возникающие при переборе элементов покрытия $\left\{\omega^{1}, \ldots, \omega^{N}\right\}$, по сути дела исчерпываются двумя упомянутыми.

\section{Список литературы}

1. Friedrichs K. O. On the boundary value problems of the theory of elasticity and Korn's inequality // Ann. Math. 1947. V. 48. P. 441-471.

2. Nečas J. Les méthodes directes en théorie des equations elliptiques. Paris: Masson, 1967.

3. Дюво Г., Лионс Ж.-Л. Неравенства в механике и физике. М.: Наука, 1980.

4. Кондратьев B. A., Олейник O. A. Краевые задачи для системы теории упругости в неограниченных областях. Неравенство Корна // УМН. 1988. Т. 43. № 5. С. 55-98.

5. Morgenstern D. Herleitung der Plattentheorie aus der dreidimensionalen Elastizitätstheorie // Arch. Rational Mech. Anal. 1959. V. 4. № 2. P. 145-152.

6. Шойхет Б. А. Об асимптотически точных уравнениях тонких плит сложной структуры // Прикладная матем. и мех. 1973. Т. 37. № 5. С. 914-924.

7. Ciarlet P. G., Kesavan S. Two-dimensional approximations of three-dimensional eigenvalue problems in plate theory // Comput. Methods Appl. Mech. Engrg. 1981. V. 26. P. $145-172$.

8. Destuynder $P$. Comparaison entre les modèles tridimensionnels et bidimensionnels de plaques en élasticité // RAIRO Modél. Math. Anal. Numér. 1981. V. 15. P. 331-369.

9. Леора С. Н., Назаров С. А., Проскура А. В. Вьвод предельных уравнений для эллиптических краевых задач в тонких областях при помощи ЭВМ // ЖВМ и МФ. 1986. Т. 26. № 7. C. $1032-1048$. 
10. Destuynder P. , Gruais I. Error estimation for the linear three-dimensional elastic plate model // Asymptotic methods for elastic structures. Berlin: Walter de Gruyter, 1995. P. $75-88$.

11. Friedrichs K. O., Dressler R.F. A boundary-layer theory for elastic plates // Comm. Pure Appl. Math. 1961. V. 14. № 1. P. 1-33.

12. Гольденвейзер $A$. Л. Построение приближенной теории изгиба пластинки методом асимптотического интегрирования уравнений теории упругости // Прикладная матем. и мех. 1962. Т. 26. № 4. С. 668-686.

13. Гольденвейзер $A$. Л., Колос $A$. В. К построению двумерных уравнений упругих тонких пластин // Прикладная матем. и мех. 1965. Т. 29. № 1. С. 141-161.

14. Назаров C. А. Структура решений эллиптических краевых задач в тонких областях // Вестник ЛГУ. 1982. № 7. С. 65-68.

15. Назаров С. А. Введение в асимптотические методы теории упругости. Л.: Изд-во ЛГУ, 1983.

16. Gregory R. D., Wan F. V. M. Decaying states of plane strain in a semi-infinite strip and boundary conditions for plate theory // J. Elasticity. 1984. V. 14. № 1. P. 27-64.

17. Зорин И. С., Назаров С. А. Краевой эффект при изгибе тонкой трехмерной пластины // Прикладная матем. и мех. 1989. Т. 53. №4. С. 642-650.

18. Назаров C. А. Асимптотика решения задачи Навье-Стокса о течении тонкого слоя жидкости // Сиб. матем. журн. 1990. Т. 31. № 2. С. 131-144.

19. Dauge M., Gruais I. Développement asymptotique d'ordre arbitraire pour une plaque élastique mince encastrée // C. R. Acad. Sci. Paris. Sér. I Math. 1995. V. 321. P. 375-380.

20. Mazja W. G., Nasarow S. A., Plamenewski B.A. Asymptotiche Theorie elliptischer Randwertaufgaben in singulär gestörten Gebieten. V. 1, 2. Berlin: Akademie-Verlag, 1991.

21. Nazarov S. A., Plamenevsky B.A. Elliptic problems in domains with piecewise smooth boundaries. Berlin: Walter de Gruyter, 1994.

22. Назаров С. А. Общая схема осреднения самосопряженных эллиптических систем в многомерных областях, в том числе тонких // Алгебра и анализ. 1995. Т. 7. № 5. С. 1-92.

23. Caillerie D. Thin elastic and periodic plates // Math. Methods Appl. Sci. 1984. V. 2. P. 251-270.

24. Панасенко Г. П., Резцов М.В. Осреднение трехмерной задачи теории упругости в неоднородных пластинах // Докл. АН СССР. 1987. Т. 294. № 5. С. 1061-1065.

25. Кондратьев B. A., Олейник О. A. О зависимости констант в неравенстве Корна от параметров, характеризующих геометрию области // УМН. 1989. Т. 44. №6. С. 157-158.

26. Назаров С.А. Неравенства Корна, асимптотически точные для тонких областей // Вестник СПбГУ. 1992. № 8. С. 19-24.

27. Cioranescu D., Oleinik O. A., Tronel G. Korn's inequalities for frame type structures and junctions with sharp estimates for the constants // Asymptotic Anal. 1994. V. 8. P. 1-14.

28. Амбариумян С. А. Теория анизотропных пластин. М.: Наука, 1987.

29. Sanchez-Palencia E. Passage à la limite de l'élasticité tridimensionelle à la théorie asymptotique des coques minces // C. R. Acad. Sci. Paris. Sér. II. 1990. V. 311. № 8. P. 909-916.

30. Зорин И. С. Операторное представление системы Ламе и предельные краевые задачи теории тонких плит // Вестник ЛГУ. 1987. № 22. С. 108.

31. Назаров C. A. Обоснование асимптотической теории тонких стержней. Интегральные и поточечные опенки // Проблемы матем. анализа. Т. 17. СПб: Изд-во СПбГУ, 1997. C. $101-152$.

32. Маховер E. В. Изгиб пластинки переменной толщины с острым краем // Ученые записки ЛГПИ. 1957. Т. 17. № 2. С. 28-39.

33. Михлин С. Г. Вариационные методы в математической физике. М.: Наука, 1970.

34. Назаров C.A. Асимптотика решений эллиптических уравнений в тонких областях с кусочно гладкой границей // Дифференциальные уравнения и их применения. Т. 33. Вильнюс: Изд-во АН ЛитССР, 1982. С. 62-83.

35. Назаров C. A., Слуцкий A.C. Асимптотика на бесконечности решений задач теории упругости в плоских параболических областях // Проблемы матем. анализа. Т. 15. СПб: Изд-во СПбГУ, 1995. С. 162-200. 
36. Назаров C.A., Слуцжий A.C. Принцип Сен-Венана для параболоидальных упругих тел // Проблемы матем. анализа. Т. 18. СПб: Изд-во СПбГУ, 1998. С. 109-156.

37. Busse S., Ciarlet P. G., Miara B. Coques "faiblement courbées" en coordonnées curvilignes // C. R. Acad. Sci. Paris. Sér. I Math. 1996. V. 322. P. 1093-1098.

38. Busse S., Ciarlet P. G., Miara B. Justification d'un modèle linèaire bi-dimensionnel de coques "faiblement courbées" en coordonnées curvilignes // RAIRO Modél. Math. Anal. Numér. 1997. V. 31. № 3. P. 409-434.

39. Назаров С.A. Самосопряженные эллиптические краевые задачи. Полиномиалное свойство и формально положительные операторы // Проблемы матем. анализа. Т. 16. СПб: Изд-во СПбГУ, 1997. С. 167-192.

40. Зорин И. С., Назаров С. А. Двучленная асимптотика решения задачи о продольной дефформации пластины, защемленной по краю // Вычислит. механика деформируемого твердого тела. 1991. Т. 2. С. 10-21.

41. Назаров С.A., Семенов Б. Н. Об асимптотике решений задач изгиба пластин с разрьвньпи нагрузками // Прикладная механика. Т. 5. Л.: Изд-во ЛГУ, 1981. С. 135-145.

42. Назаров С.A. Упругие емкость и поляризация дефекта в упругом слое // Изв. АН СССР. Механика твердого тела. 1990. № 5. С. 57-65.

43. Назаров С.A. Проявление пространственной структуры поля напряжений в окрестности угловой точки тонкой пластины // Прикладная матем. и мех. 1991. Т. 55. № 4 . C. $653-661$.

44. Алдошина И. А., Назаров С. А. Асимптотически точные условия сопряжения на стыке пластин с сильно различающимися характеристиками // Прикладная матем. и мех. 1998. T. 62. № 2. C. 272-282.

45. Назаров C. А. Несамосопряженные эллиптические задачи с полиномиальным свойством в областях, имеющих цилиндрические выходы на бесконечность // Записки научн. сем. ПОМИ. 1997. Т. 249. С. 212-231.

46. Вишик М.И., Грушин В. В. Краевые задачи для эллиптических уравнений высших порядков // Матем. сб. 1969. Т. 79. №1. С. 3-36.

47. Ильин A. М. Краевая задача для эллиптических уравнений второго порядка в области с щелью. І. Двумерный случай // Матем. сб. 1976. Т. 99. № 4. С. 514-537.

48. Kondratiev V.A., Oleinik O. A. Korn's type inequalities for a class of unbounded domains and applications to boundary-value problems in elasticity // Elasticity. Mathematical methods and application. The Ian N. Sneddon 70th Birthday Vol., 1990. P. 211-233.

49. Назаров С. А. Весовые неравенства Корна на параболоидальных областях // Матем. заметки. 1997. Т. 6. № 5. С. 751-765.

50. Ван-Дайк М. Методы возмущений в механике жидкости. М.: Мир, 1967.

51. Ильин A. М. Согласование асимптотических разложений решений эллиптических задач. М.: Наука, 1989.

Санкт-Петербургский государственный университет

Поступила в редакцию 25.01 .1999 